\title{
Finite-energy Landau liquid theory for the one-dimensional Hubbard model: Pseudoparticle energy bands and degree of localization/delocalization
}

\author{
J. M. P. Carmelo \\ GCEP-Center of Physics, University of Minho, Campus Gualtar, P-4710-057 Braga, Portugal \\ P. D. Sacramento \\ Departamento de Física and CFIF, Instituto Superior Técnico, P-1049-001 Lisboa, Portugal \\ (Received 22 July 2002; revised manuscript received 23 December 2002; published 13 August 2003)
}

\begin{abstract}
In this paper we consider the one-dimensional Hubbard model and study the deviations from the groundstate values of double occupation which result from creation or annihilation of holons, spinons, and pseudoparticles. These quantum objects are such that all energy eigenstates are described by their occupancy configurations. The band-momentum dependence of the obtained double-occupation spectra provides important information on the degree of localization/delocalization of the real-space lattice electron site distribution configurations associated with the pseudoparticles. We also study the band momentum, on-site electronic repulsion, and electronic density dependence of the pseudoparticle energy bands. The shape of these bands plays an important role in the finite-energy spectral properties of the model. Such a shape defines the form of the lines in the momentum-energy/frequency plane where the peaks and edges of the one-electron and twoelectron spectral weight of physical operators are located. Our findings are useful for the study of the oneelectron and two-electron spectral-weight distribution of physical operators.
\end{abstract}

DOI: 10.1103/PhysRevB.68.085104

\section{INTRODUCTION}

Recently, there has been a renewed experimental interest in the exotic one-electron and two-electron spectral properties of quasi-one-dimensional materials. ${ }^{1-20}$ Some of these experimental studies observed unusual finite-energy/ frequency spectral properties, which are far from being well understood. For values of the excitation energy larger than the transfer integrals associated with electron hopping between the chains, the one-dimensional (1D) Hubbard model $^{21-25}$ is expected to provide a good description of the physics of these materials. ${ }^{4,5,17,19}$ Moreover, recent angleresolved ultraviolet photoemission spectroscopy revealed very similar spectral fingerprints from both high- $T_{c}$ superconductors and quasi-one-dimensional compounds. ${ }^{2}$ The similarity of the ultraviolet data for these two different systems could be evidence of the occurrence of a charge-spin separation associated with holons and spinons. The anomalous temperature dependence of the spectral function could also indicate a dimensional crossover., ${ }^{2,26-28}$ The results of Refs. 29 and 30 also suggest that the unconventional spectral properties observed in two-dimensional (2D) materials could have a 1D origin. Thus the holons and spinons could play an important role in spectral properties of both 1D and 2D lowdimensional materials.

The present study is related to both the investigations of Refs. 31 and 32. In Ref. 31 the nonperturbative organization of the $N$ electrons and $N^{h}=\left[2 N_{a}-N\right]$ electronic holes, which results from the many-electron interactions, was studied. In that paper it was found that all energy eigenstates of the model can be described in terms of occupancy configurations of $\sigma_{c}= \pm 1 / 2$ holons, $\sigma_{s}= \pm 1 / 2$ spinons, and $c$ pseudoparticles where $\sigma_{c}$ and $\sigma_{s}$ are $\eta$-spin and spin projections, respectively. Moreover, the $c, \nu$ pseudoparticles (and $s, \nu$ pseudoparticles) associated with Takahasi's charge (and spin) ideal string excitations of length $\nu$ (Refs. 22 and 31) were found to be $\eta$-spin singlet $2 \nu$-holon (and spin singlet $2 \nu$-spinon) composite quantum objects. (The $c$ and $\alpha, \nu$ pseudoparticles are obtained naturally and directly from analysis of the Bethe-ansatz solution. ${ }^{31}$ ) In that reference, the $\pm 1 / 2$ holons (and $\pm 1 / 2$ spinons) which are not part of such $2 \nu$-holon composite $c, \nu$ pseudoparticles (and $2 \nu$-spinon composite $s, \nu$ pseudoparticles) were called $\pm 1 / 2 \mathrm{C}$. N. Yang (Yang) holons [and $\pm 1 / 2$ Heilmann and Lieb (HL) spinons]. ${ }^{31}$ Following the notation of Ref. 31, here we call $N_{c}$ and $N_{\alpha, \nu}$ the numbers of $c$ pseudoparticles and $\alpha, \nu$ pseudoparticles belonging to branches $\alpha=c, s$ and $\nu$ $=1,2,3, \ldots$, respectively. On the other hand, it was found in Ref. 31 that the charge excitations associated with the $c$ pseudoparticles are independent of the $\eta$-spin-holon and spin-spinon excitations. Moreover, the results of Ref. 32 reveal that the above quantum objects can be expressed in terms of rotated electrons. According to these results the first step of the nonperturbative diagonalization of the model corresponds to a mere unitary rotation in Hilbert space which maps the electrons onto rotated electrons. For rotated electrons double occupation is a good quantum number for all values of the on-site repulsion. Interestingly, such a rotation corresponds to a unitary transformation previously introduced by Harris and Lange. ${ }^{33}$ Such a transformation is shown in Ref. 32 to play a key role in the expression of the holon and spinon number operators in terms of electronic operators for the whole parameter space of the model. The $N_{c} c$ pseudoparticles (and $N_{c}$ spinons) describe the charge part (and spin part) of the $N_{c}$ rotated electrons which singly occupy lattice sites. Thus the charge and spin degrees of freedom of such a $N_{c}$-rotated-electron fluid separate. For each $c$ pseudoparticle there is a chargeon ${ }^{31}$ and a rotatedelectronic hole. Such a chargeon corresponds to the charge part of a rotated electron which singly occupies a site. We 
note that the numbers $N_{c},\left[N-N_{c}\right] / 2$, and $\left[N^{h}-N_{c}\right] / 2$ equal the number of rotated-electron singly occupied sites, doubly occupied sites, and empty sites, respectively. The nonperturbative organization of the electronic degrees of freedom also includes a $\left[N-N_{c}\right]$-rotated-electron fluid associated with the rotated-electron pairs occupying $\left[N-N_{c}\right] / 2$ sites. Each such a doubly occupied site corresponds to a spin singlet rotatedelectron pair which is nothing but a $-1 / 2$ holon. Such a two-fluid picture shows some formal analogies to those which characterize a superconductor or a superfluid. Finally, the rotated-electron $\left[N^{h}-N_{c}\right] / 2$ empty sites are nothing but the $+1 / 2$ holons.

The main goal of this paper is the study of the $c$-pseudoparticle; $\alpha, \nu$-pseudoparticle; $-1 / 2$ Yang holon; and $-1 / 2$ HL spinon double-occupation spectra. These functions are evaluated by use of the Hellmann-Feynman theorem. ${ }^{34,35}$ In the case of the pseudoparticles we find band-momentumdependent double-occupation spectra. Analysis of these spectra provides important information about the localization/ delocalization degree of the real-space lattice electronic occupancy configurations which describe the pseudoparticles. We find that for some of the pseudoparticle branches such a degree of localization/delocalization is strongly dependent on the value of the pseudoparticle band momentum. Our analysis leads to a double-occupation functional whose coefficients are the double-occupation spectra. This study confirms that the complex electron site distribution configurations of the real-space lattice which describe the energy eigenstates are dependent on the value of $U / t$. We also consider $U / t \rightarrow \infty$ electron double-occupation selection rules. These rules are used in Ref. 32 to find exact rotated-electron selection rules which provide for all values of the on-site electronic repulsion the number of holons and spinons of the final states which contribute most significantly to fewelectron correlation functions. These selection rules imply that a large number of matrix elements between energy eigenstates are vanishing. This simplifies the derivation of general expressions for correlation functions at finite excitation energy. This problem is studied in Refs. 36 and 37.

In addition, we study the band momentum, on-site electronic repulsion $U$, and electronic density $n$ dependence of the pseudoparticle energy bands. Such pseudoparticle energy bands are the coefficients of the first-order term of the finiteenergy Landau-liquid functional. That functional was obtained in Ref. 38 by expanding the $c$-pseudoparticle; $c, \nu$-pseudoparticle; and $s, \nu$-pseudoparticle band-momentum distribution functions around their ground-state values. This is similar to the case of the quasiparticle energy functional of Fermi-liquid theory. ${ }^{39,40}$ The pseudoparticle bands are the basic blocks of the energy spectra of the elementary excitations of the 1D Hubbard model, such as, for example, the ones studied in Refs. 41-46.

The finite-energy theory constructed here and in Refs. 31, 32,36 , and 37 is applied elsewhere to the study of the finiteenergy/frequency one-electron and two-electron spectral properties of quasi-one-dimensional materials. Reference 47 is an application of a preliminary version of our finite-energy theory. The evaluation of the spectral functions of the model reveals that the one-electron and two-electron spectral weight of physical operators contains peaks and/or edges which are located on well-defined lines in the momentum $k$ and energy/frequency $\omega$ plane. Importantly, the shape of these peak and edge lines is fully defined by the bandmomentum dependence of the pseudoparticle energy bands studied in this paper. Moreover, the expressions of the finiteenergy correlation functions involve these pseudoparticle bands. ${ }^{37}$ Therefore, our investigation is important for the study of the one-electron and two-electron spectral-weight distribution of physical operators. The study of the localization-delocalization degree of the real-space lattice electronic occupancy configurations which describe the pseudoparticles also contributes to important information about the physics behind these elementary quantum objects. Such a study also provides information about the electron site distribution configurations of the real-space lattice which describe the energy eigenstates.

The paper is organized as follows: In Sec. II we introduce the 1D Hubbard model. The concepts of $c$-pseudoparticle, holon, and spinon (CPHS) ensemble space and subspace are introduced in Sec. III. In Sec. IV the double-occupation functional and its pseudoparticle, $-1 / 2$ Yang holon, and $-1 / 2$ HL spinon spectra are obtained and discussed. In this section we also consider $U / t \rightarrow \infty$ selection rules in the values of double-occupation deviations which result from $\mathcal{N}$-electron excitations. In Sec. V we study the band-momentum dependence of the energy pseudoparticle bands. Finally, the concluding remarks are presented in Sec. VI.

\section{THE 1D HUBBARD MODEL}

In a chemical potential $\mu$ and magnetic field $H$ the 1D Hubbard Hamiltonian can be written as

$$
\hat{H}=\hat{H}_{S O(4)}+\sum_{\alpha=c, s} \mu_{\alpha} \hat{S}_{z}^{\alpha},
$$

where the Hamiltonian

$$
\hat{H}_{S O(4)}=\hat{H}_{H}-(U / 2) \hat{N}+(U / 4) N_{a}
$$

has $\mathrm{SO}(4)$ symmetry $^{48,49}$ and

$$
\hat{H}_{H}=\hat{T}+U \hat{D}
$$

is the basic 1D Hubbard model. On the right-hand side of Eq. (3),

$$
\hat{T}=-t \sum_{j, \sigma}\left[c_{j, \sigma}^{\dagger} c_{j+1, \sigma}+\text { H. c. }\right]
$$

is the kinetic-energy operator, $t$ is the first-neighbor transfer integral, and $U$ is the on-site repulsion associated with electronic double occupation. The operator

$$
\hat{D}=\sum_{j} \hat{n}_{j, \uparrow} \hat{n}_{j, \downarrow}
$$

counts the number of electron doubly occupied sites and the operator

$$
\hat{n}_{j, \sigma}=c_{j, \sigma}^{\dagger} c_{j, \sigma}
$$


counts the number of spin $\sigma$ electrons at site $j$. The operators $c_{j, \sigma}^{\dagger}$ and $c_{j, \sigma}$ which appear in the above equations are the spin $\sigma$ electron creation and annihilation operators at site $j$, respectively. Moreover, on the right-hand side of Eq. (1), $\mu_{c}=2 \mu, \mu_{s}=2 \mu_{0} H, \mu_{0}$ is the Bohr magneton, and

$$
\hat{S}_{z}^{c}=-\frac{1}{2}\left[N_{a}-\hat{N}\right], \quad \hat{S}_{z}^{s}=-\frac{1}{2}\left[\hat{N}_{\uparrow}-\hat{N}_{\downarrow}\right]
$$

are the diagonal generators of the SU(2) $\eta$-spin $S^{c}$ and spin $S^{s}$ algebras, ${ }^{48,49}$ respectively. On the right-hand side of Eqs. (2) and (7) the number of lattice sites $N_{a}$ is even and large, $N_{a} / 2$ is odd, $\hat{N}=\Sigma_{\sigma} \hat{N}_{\sigma}$, and $\hat{N}_{\sigma}=\Sigma_{j} \hat{n}_{j, \sigma}$.

There are $N_{\uparrow}$ spin-up electrons and $N_{\downarrow}$ spin-down electrons in the chain of $N_{a}$ sites and with lattice constant $a$ associated with the model (1). We assume periodic boundary conditions and employ units such that $a=\hbar=1$. When $N_{\sigma}$ is odd the Fermi momenta are given by $k_{F \sigma}^{ \pm}= \pm\left[k_{F \sigma}\right.$ $\left.-\left(\pi / N_{a}\right)\right]$, where

$$
k_{F \sigma}=\frac{\pi N_{\sigma}}{N_{a}} .
$$

$11 ;-3 \mathrm{q}$ When $N_{\sigma}$ is even the Fermi momenta are given by $k_{F \sigma}^{+}=k_{F \sigma}$ and $k_{F \sigma}^{-}=-\left[k_{F \sigma}-\left(2 \pi / N_{a}\right)\right]$ or by $k_{F \sigma}^{+}=\left[k_{F \sigma}\right.$ $\left.-\left(2 \pi / N_{a}\right)\right]$ and $k_{F \sigma}^{-}=-k_{F \sigma}$. Often we can ignore the $1 / N_{a}$ corrections of these expressions and consider $k_{F \sigma}^{ \pm} \simeq \pm k_{F \sigma}$ $= \pm \pi n_{\sigma}$ and $k_{F}=\left[k_{F \uparrow}+k_{F \downarrow}\right] / 2=\pi n / 2$, where $n_{\sigma}=N_{\sigma} / N_{a}$ and $n=N / N_{a}$. The electronic density reads $n=n_{\uparrow}+n_{\downarrow}$, and the spin density is given by $m=n_{\uparrow}-n_{\downarrow}$. This paper uses the same notations as those in Ref. 31 and often uses and refers to the equations introduced in that paper. For instance, $M_{c, \pm 1 / 2}\left(\right.$ and $\left.M_{s, \pm 1 / 2}\right)$ denotes the number of $\pm 1 / 2$ holons (and $\pm 1 / 2$ spinons), whereas $L_{c, \pm 1 / 2}$ (and $L_{s, \pm 1 / 2}$ ) denotes the number of $\pm 1 / 2$ Yang holons (and $\pm 1 / 2$ HL spinons).

The Hamiltonian $\hat{H}_{S O(4)}$ defined in Eq. (2) commutes with the six generators of the $\eta$-spin $S_{c}$ and spin $S_{s}$ algebras, ${ }^{48,49}$ their off-diagonal generators reading

$$
\hat{S}_{+}^{c}=\sum_{j}(-1)^{j} c_{j, \downarrow}^{\dagger} c_{j, \uparrow}^{\dagger}, \quad \hat{S}_{-}^{c}=\sum_{j}(-1)^{j} c_{j, \uparrow} c_{j, \downarrow},
$$

and

$$
\hat{S}_{+}^{s}=\sum_{j} c_{j, \downarrow}^{\dagger} c_{j, \uparrow}, \quad \hat{S}_{-}^{s}=\sum_{j} c_{j, \uparrow}^{\dagger} c_{j, \downarrow} .
$$

The Bethe-ansatz solvability of the 1D Hubbard model is restricted to the Hilbert subspace spanned by the lowestweight states (LWSs) of the $\eta$ spin and spin algebras, i.e., such that $S^{\alpha}=-S_{z}^{\alpha}$. 50

\section{CPHS-ENSEMBLE SPACES AND GROUND-STATE DISTRIBUTIONS AND RAPIDITIES}

The studies of Ref. 31 considered Hilbert subspaces associated with fixed values of $\eta$-spin $S_{c}$, spin $S_{s}$, holon number $M_{c}$, and spinon number $M_{s}$. That was the choice suitable for the investigations on $\eta$-spin-holon, spin-spinon, and charge $c$-pseudoparticle separation of the excitations of the 1D Hubbard model at all energy scales. However, fixing $S_{c}$,
$S_{s}, M_{c}$, and $M_{s}$ does not fix the $N_{\uparrow}$ - and $N_{\downarrow}$-electron numbers.

In proceeding sections of this paper and in Refs. 32, 36, and 37 transitions from a ground state with given spin $\sigma$ electron numbers to excited states are studied. Thus it is convenient for these studies to consider subspaces with fixed number of spin $\sigma$ electrons. From the use of Eq. (7) and Eq. (35) of Ref. 31, the $N_{\uparrow}$ - and $N_{\downarrow}$-electron numbers can be expressed in terms of the $M_{c,+1 / 2}$ and $M_{c,-1 / 2}$ holon numbers and $M_{s,+1 / 2}$ and $M_{s,-1 / 2}$ spinon numbers as follows,

$$
N_{\uparrow}=\frac{1}{2}\left\{N_{a}-\left(M_{c,+1 / 2}-M_{c,-1 / 2}\right)+\left(M_{s,+1 / 2}-M_{s,-1 / 2}\right)\right\}
$$

and

$$
N_{\downarrow}=\frac{1}{2}\left\{N_{a}-\left(M_{c,+1 / 2}-M_{c,-1 / 2}\right)-\left(M_{s,+1 / 2}-M_{s,-1 / 2}\right)\right\},
$$

respectively. We find from the use of Eq. (32) of Ref. 31 that the $M_{c,+1 / 2}$ holon and $M_{s,+1 / 2}$ spinon numbers are exclusive functions of the $N_{c} c$-pseudoparticle number and $M_{c,-1 / 2}$ holon and $M_{s,-1 / 2}$ spinon numbers and can be written as

$$
M_{c,+1 / 2}=N_{a}-N_{c}-M_{c,-1 / 2}, \quad M_{s,+1 / 2}=N_{c}-M_{s,-1 / 2} .
$$

It is convenient for our studies to express the $N_{\uparrow}$ - and $N_{\downarrow}$-electron numbers in terms of the $N_{c} c$-pseudoparticle numbers, $M_{c,-1 / 2}$ holon numbers, and $M_{s,-1 / 2}$ spinon numbers, with the result

$$
N_{\uparrow}=N_{c}+M_{c,-1 / 2}-M_{s,-1 / 2},
$$

and

$$
N_{\downarrow}=M_{c,-1 / 2}+M_{s,-1 / 2},
$$

respectively.

We call an electron ensemble space a Hilbert subspace spanned by all states with fixed values for the $N_{\uparrow}$ - and $N_{\downarrow}$-electron numbers. Furthermore, we call a CPHSensemble space, where CPHS stands for $c$ pseudoparticle, $-1 / 2$ holon, and $-1 / 2$ spinon, a Hilbert subspace spanned by all states with fixed values for the numbers $N_{c}, M_{c,-1 / 2}$, and $M_{s,-1 / 2}$ of $c$ pseudoparticles, $-1 / 2$ holons, and $-1 / 2$ spinons, respectively. It follows from Eqs. (14) and (15) that, in general, an electron ensemble space contains several CPHS-ensemble spaces. This means that different choices of the numbers $N_{c}, M_{c,-1 / 2}$, and $M_{s,-1 / 2}$ can lead to the same electron numbers $N_{\uparrow}$ and $N_{\downarrow}$. In contrast, according to Eqs. (14) and (15), a given choice of $N_{c}, M_{c,-1 / 2}$, and $M_{s,-1 / 2}$ always corresponds to an unique choice for the $N_{\uparrow}$ and $N_{\downarrow}$ values of the electron numbers.

Each CPHS-ensemble space contains several subspaces with different numbers $L_{c,-1 / 2}$ of $-1 / 2$ Yang holons, $L_{s,-1 / 2}$ of $-1 / 2 \mathrm{HL}$ spinons, and sets of numbers $\left\{N_{c, \nu}\right\}$ and $\left\{N_{s, \nu}\right\}$ of $c, \nu$ and $s, \nu$ pseudoparticles, respectively, corresponding to different $\nu=1,2,3, \ldots$ branches. According to the results 
of Ref. 31, the fixed values of each subspace obey the constraints imposed by the following equations,

$$
\begin{aligned}
M_{c,-1 / 2}= & L_{c,-1 / 2}+\sum_{\nu=1}^{\infty} \nu N_{c, \nu} ; \quad M_{s,-1 / 2}=L_{s,-1 / 2} \\
& +\sum_{\nu=1}^{\infty} \nu N_{s, \nu} .
\end{aligned}
$$

We call a CPHS-ensemble subspace a Hilbert subspace spanned by all states with fixed values for the numbers $N_{c}$, $L_{c,-1 / 2}$, and $L_{s,-1 / 2}$ and for the sets of numbers $\left\{N_{c, v}\right\}$ and $\left\{N_{s, \nu}\right\}$ corresponding to $\nu=1,2,3, \ldots$ branches. It follows from Eqs. (16) that in general, a CPHS-ensemble space contains several CPHS-ensemble subspaces. This means that different choices of the values for $L_{c,-1 / 2}$ and $L_{s,-1 / 2}$ and sets of numbers $\left\{N_{c, \nu}\right\}$ and $\left\{N_{s, \nu}\right\}$ corresponding to $\nu=1,2,3, \ldots$. branches can lead to the same values for $M_{c,-1 / 2}$ and $M_{s,-1 / 2}$. In contrast, a given choice of the values of $N_{c}$, $L_{c,-1 / 2}$, and $L_{s,-1 / 2}$ and sets $\left\{N_{c, \nu}\right\}$ and $\left\{N_{s, \nu}\right\}$ corresponding to $\nu=1,2,3, \ldots$ branches always corresponds to unique choice of the values of $N_{c}, M_{c,-1 / 2}$, and $M_{s,-1 / 2}$.

Finally, we note that one does not need to provide the values of $M_{c,+1 / 2}$ and $M_{s,+1 / 2}$ in order to specify a CPHSensemble space. These numbers are given by Eq. (13) and are not independent. Furthermore, from the combination of Eqs. (30), (32), and (34) of Ref. 31 one finds that

$$
\begin{gathered}
L_{c,+1 / 2}=N_{a}-N_{c}-2 \sum_{\nu=1}^{\infty} \nu N_{c, \nu}-L_{c,-1 / 2} ; \\
L_{s,+1 / 2}=N_{c}-2 \sum_{\nu=1}^{\infty} \nu N_{s, \nu}-L_{s,-1 / 2} .
\end{gathered}
$$

Thus $L_{c,+1 / 2}$ and $L_{s,+1 / 2}$ are not independent and one does not need to provide these values in order to specify a CPHSensemble subspace. Therefore, often we do not consider the values of the holon numbers $M_{c,+1 / 2}$ and $L_{c,+1 / 2}$ and of the spinon numbers $M_{s,+1 / 2}$ and $L_{s,+1 / 2}$ in the expressions considered below.

According to the studies of Ref. 31, our choice of using the numbers $N_{c}$ and $M_{c,-1 / 2}$ to label the charge sector of a CPHS-ensemble space corresponds to a description of charge transport in terms of electrons. In this case the elementary charge carriers are the $N_{c}$ chargeons and $M_{c,-1 / 2}-1 / 2$ holons, which carry charges $-e$ and $-2 e$, respectively. Here $-e$ denotes the charge of the electron. The alternative use of the numbers $N_{c}$ and $M_{c,+1 / 2}$ to define the charge sector of a CPHS-ensemble space would correspond to a description of charge transport in terms of electronic holes. In this case the elementary charge carriers would be the $N_{c}$-rotated-electronic holes and $M_{c,+1 / 2}+1 / 2$ holons, which carry charges $+e$ and $+2 e$, respectively. ${ }^{31}$ Also the transport of spin has two alternative descriptions which correspond to the $-1 / 2$ spinons and $+1 / 2$ spinons, respectively. Here we have chosen to label the spin sector of the CPHSensemble spaces by the number $M_{s,-1 / 2}$ of $-1 / 2$ spinons.
The finite-energy Landau-liquid functional discussed in Ref. 31 and used in the ensuing sections corresponds to the CPHS-ensemble subspace description in terms of the values of the numbers of $-1 / 2$ Yang holons and $-1 / 2$ HL spinons and of the values of the numbers of $c$ pseudoparticles at band momentum $q$ and of $\alpha, \nu$ pseudoparticles belonging to $\alpha$ $=c, s$ and $\nu=1,2,3, \ldots$ branches at band momentum $q$. According to the studies of Ref. 31, such a choice corresponds again to the description of charge transport in terms of electrons. In this case the $c, \nu$ pseudoparticle carries charge $-2 \nu e$.

\section{ELECTRON DOUBLE OCCUPATION OF THE PSEUDOPARTICLES, YANG HOLONS, AND HL SPINONS}

Let us denote the number of doubly occupied sites by $D$. By the number of doubly occupied sites we mean here the expectation value of the operator (5),

$$
D \equiv\langle\hat{D}\rangle=\sum_{j}\left\langle c_{j, \uparrow}^{\dagger} c_{j, \uparrow} c_{j, \downarrow}^{\dagger} c_{j, \downarrow}\right\rangle .
$$

Another useful quantity is the kinetic energy $T$, which we define as the expectation value of the operator (4),

$$
T \equiv-t \sum_{j, \sigma}\left[\left\langle c_{j, \sigma}^{\dagger} c_{j+1, \sigma}\right\rangle+\left\langle c_{j+1, \sigma}^{\dagger} c_{j, \sigma}\right\rangle\right] .
$$

Let $|\psi\rangle$ be an energy eigenstate of the Hamiltonian (3) of energy $E_{H}=\left\langle\psi\left|\hat{H}_{H}\right| \psi\right\rangle$ given by the general expression

$$
\begin{aligned}
E_{H}= & -2 t \frac{N_{a}}{2 \pi} \int_{q_{c}^{-}}^{q_{c}^{+}} d q N_{c}(q) \cos k(q) \\
& +4 t \frac{N_{a}}{2 \pi} \sum_{\nu=1}^{\infty} \int_{q_{c, \nu}^{-}}^{q_{c, \nu}^{+}} d q N_{c, \nu}(q) \\
& \times \operatorname{Re}\left\{\sqrt{1-\left[\Lambda_{c, \nu}(q)+i \nu U / 4 t\right]^{2}}\right\}+U L_{c,-1 / 2},
\end{aligned}
$$

where $k(q)$ and $\Lambda_{c, \nu}(q)$ are the rapidity functionals defined by the integral in Eqs. (13)-(16) of Ref. 31 and $N_{c}(q)$ and $N_{c, \nu}(q)$ are pseudoparticle band-momentum distribution functions also defined therein. The expressions of the bandmomentum limiting values of the integrals on the right-hand side of Eq. (20) are given in Eqs. (B14)-(B17) of Ref. 31. This energy expression is obtained by combining the energy expression provided by the Bethe-ansatz solution ${ }^{22,38,46}$ with the $\mathrm{SO}(4)$ symmetry of the model and the holon and spinon descriptions introduced in Ref. 31. Note that such a general energy expression refers to the whole Hilbert space of the 1D Hubbard model. The term involving the number of $-1 / 2$ Yang holons vanishes in the case of the LWSs of the SU(2) $\eta$-spin algebra associated with the Bethe-ansatz solution and is not provided by such a solution.

According to the Hellmann-Feynman theorem, ${ }^{34,35}$ the electron double occupation $D$, and kinetic energy $T$, associated with the above energy eigenstate, are given by 


$$
D \equiv\left\langle\psi\left|\frac{\partial \hat{H}_{H}}{\partial U}\right| \psi\right\rangle=\frac{\partial\left\langle\psi\left|\hat{H}_{H}\right| \psi\right\rangle}{\partial U}=\frac{\partial E_{H}}{\partial U}
$$

and

$$
T \equiv\left\langle\psi\left|t \frac{\partial \hat{H}_{H}}{\partial t}\right| \psi\right\rangle=t \frac{\partial\left\langle\psi\left|\hat{H}_{H}\right| \psi\right\rangle}{\partial t}=t \frac{\partial E_{H}}{\partial t},
$$

respectively, where $\hat{H}_{H}$ is the Hamiltonian defined in Eq. (3) and the energy $E_{H}$ is given by Eq. (20).

The use of expression (21) in the evaluation of the electron double-occupation spectrum associated with creation of a $c$ pseudoparticle; $\alpha, \nu$ pseudoparticle; $-1 / 2$ Yang holon; or $-1 / 2 \mathrm{HL}$ spinon involves the pseudoparticle Landau-liquid energy functional. This functional was introduced in Ref. 38 and expressed in terms of holon and spinon numbers in Ref. 31.

\section{A. The pseudoparticle, holon, and spinon Landau-liquid energy functional}

The energy Landau-liquid functional introduced in Ref. 38 is generated by expanding the $c$ and $\alpha, \nu$-pseudoparticle band-momentum distribution functions $N_{c}(q)$ and $\left\{N_{\alpha, \nu}(q)\right\}$ where $\alpha=c, s$ and $\nu=1,2,3, \ldots$. Such an expansion is performed around the ground-state values of these functions given in Appendix C of Ref. 31 and inserting these distributions in the energy functional $E$ associated with the Hamiltonian (1). Such an energy functional is given by

$$
E=E_{H}+\sum_{\alpha=c, s} \mu_{\alpha} S_{z}^{\alpha}+\frac{U}{2}\left[L_{c,+1 / 2}-L_{c,-1 / 2}-\frac{N_{a}}{2}\right],
$$

where $E_{H}$ is the functional (20), the parameters $\mu_{\alpha}$ are the same as in Eq. (1), and $S_{z}^{\alpha}$ are the eigenvalues of the diagonal generators defined in Eq. (7). Let us consider that the initial ground state corresponds to values of the electronic density such that $0 \leqslant n \leqslant 1$ and values of the spin density such that $0 \leqslant m \leqslant n$. Throughout this paper we consider such ground states. The studies of Ref. 38 have not expressed the energy Landau-liquid functional in terms of the deviations in the value of the $-1 / 2$ Yang holon and $-1 / 2$ HL spinon numbers. That problem was first addressed in Ref. 31. As discussed in that reference, the general excitation spectrum of interest for the problem of the finite-energy correlation functions can be written as the sum of a finite energy $\omega_{0}$ and of a gapless contribution expressed in terms of the pseudoparticle energy bands. Such a energy Landau-liquid functional reads

$$
\begin{aligned}
\Delta E= & \omega_{0}+\frac{N_{a}}{2 \pi} \int_{q_{c}^{-}}^{q_{c}^{+}} d q \epsilon_{c}(q) \Delta N_{c}(q) \\
& +\frac{N_{a}}{2 \pi} \int_{-q_{s, 1}}^{+q_{s, 1}} d q \epsilon_{s, 1}(q) \Delta N_{s, 1}(q) \\
& +\frac{N_{a}}{2 \pi} \sum_{\alpha=c, s} \sum_{\nu=1+\delta_{\alpha, s}}^{\infty} \int_{-q_{\alpha, \nu}}^{+q_{\alpha, \nu}} d q \epsilon_{\alpha, \nu}^{0}(q) \Delta N_{\alpha, \nu}(q),
\end{aligned}
$$

where $\Delta N_{c}(q), \Delta N_{c, \nu}(q)$, and $\Delta N_{s \nu}(q)$ are the pseudoparticle band-momentum distribution function deviations. The band-momentum limiting values $q_{s, 1}, q_{\alpha, \nu}$, and $q_{c}^{ \pm}$are defined in Eqs. (B14)-(B17) of Ref. 31, whereas the pseudoparticle energy bands $\epsilon_{c}(q), \epsilon_{s, 1}(q)$, and $\epsilon_{\alpha, \nu}^{0}(q)$ are defined by Eqs. (C15)-(C21) of the same reference.

The expression for the energy parameter $\omega_{0}$ on the righthand side of Eq. (24) which controls the finite-energy physics is determined by the number of $-1 / 2$ holons and $-1 / 2$ spinons (except those associated with $s, 1$ pseudoparticles) of the final states relative to the ground state. Creation of a $-1 / 2$ holon requires an amount of energy $2 \mu$ and momentum $\pi$ whereas creation of a $-1 / 2$ spinon (except those which are part of $s, 1$ pseudoparticles) requires an amount of energy $2 \mu_{0} H$ and zero momentum. Thus the energy parameter $\omega_{0}$ is a simple expression in terms of the deviations in the numbers of $-1 / 2$ holons and $-1 / 2$ spinons, which reads

$$
\omega_{0}=2 \mu \Delta M_{c,-1 / 2}+2 \mu_{0} H\left[\Delta M_{s,-1 / 2}-\Delta N_{s, 1}\right],
$$

where $\Delta M_{\alpha,-1 / 2} \equiv\left[M_{\alpha,-1 / 2}-M_{\alpha,-1 / 2}^{0}\right]$ are the deviations in the numbers of $-1 / 2$ holons $(\alpha=c)$ and of $-1 / 2$ spinons $(\alpha=s)$, and $\Delta N_{s, 1} \equiv\left[N_{s, 1}-N_{s, 1}^{0}\right]$ is the deviation from the ground-state value of the number of $s, 1$ pseudoparticles. The ground-state pseudoparticle numbers are given in Appendix C of Ref. 31.

The final states associated with the energy functional (24) belong to different CPHS-ensemble spaces, depending on the numbers of quantum objects of these states. According to the form of the general energy spectrum defined in Eqs. (24) and (25), final states belonging to CPHS-ensemble spaces with finite occupancies of $-1 / 2$ holons have finite excitation energy relative to the ground state. These states belong to CPHS-ensemble subspaces which are spanned by states with finite occupancies of $-1 / 2$ Yang holons and/or of $c, \nu$ pseudoparticles. Again following expression (25), for finite values of the spin density $m$, states belonging to CPHSensemble subspaces spanned by states with finite occupancies of $-1 / 2 \mathrm{HL}$ spinons, and/or of $s, \nu$ pseudoparticles belonging to $\nu>1$ branches, have also finite excitation energy relative to the ground state. However, most of the results of the present paper consider the limit of zero-spin density, $m$ $=0$, where the energy spectrum of these states is gapless.

On the other hand, for general values of $n$ and $m$ the low-energy Hilbert subspace corresponds to the CPHSensemble space which contains the ground state. The corresponding ground-state $c$ pseudoparticle, $-1 / 2$ holon, and $-1 / 2$ spinon numbers are given in Eqs. (C24) and (C25) of Ref. 31. Such a CPHS-ensemble space is spanned by states with finite pseudoparticle occupancy for the $c$ - and $s, 1$-pseudoparticle bands only. We recall that according to the results of Ref. 31 the $s, 1$ pseudoparticle is a composite quantum object. It is constituted of two spinons of opposite spin projection. In the studies of Refs. 51-53 the $c$ - and $s, 1$-pseudoparticle branches were referred to as $c$ pseudoparticles and $s$ pseudoparticles, respectively. The excited states contained in that CPHS-ensemble space have bandmomentum distribution functions whose occupancies are different from the ones of the $c$ and $s, 1$ ground-state distribu- 
tions provided in Eqs. (C1) and (C2), respectively, of Ref. 31. At fixed spin $\sigma$ electron numbers, the occupancies of these excited states can be generated from the ground state by pseudoparticle-pseudoparticle hole processes in the $c$ and $s, 1$ bands.

\section{B. The ground-state electron double occupation}

In the particular case of a zero-spin-density ground state, from the use of the general Eq. (21), we find

$$
D_{0}=\frac{\partial E_{G S H}}{\partial U}=\frac{N}{2}\left(\frac{n}{2}\right) f(n, U / t),
$$

where $D_{0}$ denotes the ground-state electron double occupation and $E_{G S H}$ stands for the ground-state energy

$$
\begin{aligned}
E_{G S H} & =-2 t \frac{N_{a}}{2 \pi} \int_{q_{F c}^{-}}^{q_{F c}^{+}} d q \cos k^{0}(q) \\
& =-2 t \frac{N_{a}}{2 \pi} \int_{-Q}^{Q} d k 2 \pi \rho_{c}(k) \cos k .
\end{aligned}
$$

Here the Fermi band momenta $q_{F c}^{ \pm}$are defined by Eqs. (C4)(C7) of Ref. 31 and the ground-state functions $k^{0}(q)$ and $2 \pi \rho_{c}(k)$ are studied below. This energy expression corresponds to the Hamiltonian (3) and is obtained by use of the ground-state numbers and deviations of Eqs. (C1)-(C3) and (C24) and (C25) of Ref. 31 in the general energy functional (20). In order to study the ground-state double occupation (26), let us provide useful information on the related energy (27). The ground-state function $k^{0}(q)$ on the right-hand side of Eq. (27) is related to the distribution $2 \pi \rho_{c}(k)$ considered below by the following equation,

$$
\frac{\partial k^{0}(q)}{\partial q}=\frac{1}{2 \pi \rho_{c}\left[k^{0}(q)\right]} .
$$

The function $k^{0}(q)$ is also related to the ground-state rapidity functions $\Lambda_{\alpha, \nu}^{0}(q)$ such that

$$
\frac{\partial \Lambda_{\alpha, \nu}^{0}(q)}{\partial q}=\frac{1}{2 \pi \sigma_{\alpha, \nu}\left(\Lambda_{\alpha, \nu}^{0}(q)\right)} ; \quad \alpha=c, s, \quad \nu=1,2,3, \ldots .
$$

The above functions $2 \pi \rho_{c}(k), 2 \pi \sigma_{c, \nu}(\Lambda)$, and $2 \pi \sigma_{s, \nu}(\Lambda)$ are the solutions of the following integral equations,

$$
\begin{aligned}
2 \pi \rho_{c}(k)= & +\frac{4 t \cos k}{\pi} \\
& \times \int_{-B}^{B} d \Lambda \frac{U}{U^{2}+(4 t)^{2}(\Lambda-\sin k)^{2}} 2 \pi \sigma_{s, 1}(\Lambda),
\end{aligned}
$$

$$
\begin{aligned}
2 \pi \sigma_{c, \nu}(\Lambda)= & 2 \operatorname{Re}\left[\frac{4 t}{\sqrt{(4 t)^{2}-(4 t \Lambda-i \nu U)^{2}}}\right] \\
& -\frac{4 t}{\pi} \int_{-Q}^{Q} d k \frac{\nu U}{(\nu U)^{2}+(4 t)^{2}(\sin k-\Lambda)^{2}} \\
& \times 2 \pi \rho_{c}(k),
\end{aligned}
$$

and

$$
\begin{aligned}
2 \pi \sigma_{s, \nu}(\Lambda)= & \frac{4 t}{\pi} \int_{-Q}^{Q} d k \frac{\nu U}{(\nu U)^{2}+(4 t)^{2}(\sin k-\Lambda)^{2}} 2 \pi \rho_{c}(k) \\
& -\frac{2 t}{\pi U} \int_{-B}^{B} d \Lambda^{\prime} \Theta_{1, \nu}^{[1]}\left[\frac{4 t\left(\Lambda-\Lambda^{\prime}\right)}{U}\right] 2 \pi \sigma_{s, 1}\left(\Lambda^{\prime}\right),
\end{aligned}
$$

where the expression of the function $\Theta_{1, \nu}^{[1]}(x)$ and the parameters $Q$ and $B$ are given in Eqs. (C22) and (C23) of Ref. 31. We note that $2 \pi \rho_{c}(k)$ and $2 \pi \sigma_{s, 1}(\Lambda)$ equal the functions $2 \pi \rho(k)$ and $2 \pi \sigma(\Lambda)$, respectively, introduced by Lieb and Wu. ${ }^{21}$ At half filling and spin density $m=0$ the parameters $Q$ and $B$ read $Q=\pi$ and $B=\infty$ and Eqs. (30)-(32) can be solved by Fourier transform. Use of the obtained expressions in Eq. (28) and in Eq. (29) for $\alpha, \nu=s, 1$ leads to the following expressions,

$$
q=k^{0}(q)+2 \int_{0}^{\infty} d x J_{0}(x) \frac{\sin \left[x \sin k^{0}(q)\right]}{x\left[1+e^{x U / 2 t}\right]} ; \quad|q| \leqslant \pi,
$$

and

$$
q=\int_{0}^{\infty} d x J_{0}(x) \frac{\sin \left[x \Lambda_{s, 1}^{0}(q)\right]}{x \cosh \left(\frac{x U}{4 t}\right)} ; \quad|q| \leqslant \pi / 2,
$$

where $J_{0}(x)$ and $J_{1}(x)$ are Bessel functions. These expressions define the inverse functions of $k^{0}(q)$ and $\Lambda_{s, 1}^{0}(q)$, respectively. Based on these expressions it is straightforward to find that at half filling and zero-spin density the energy (27) can be written in closed form with the result ${ }^{21}$

$$
E_{G S H}=-4 N_{a} t \int_{0}^{\infty} d x \frac{J_{0}(x) J_{1}(x)}{x\left[1+e^{x U / 2 t}\right]}
$$

One can use the obtained ground-state energy expressions in the evaluation of the electron ground-state double occupation (26). We find that in the limits of vanishing and infinite on-site repulsion $U / t$ the function $f(n, U / t)$ of Eq. (26) is given by 1 and 0 , respectively. Closed-form expressions for that function can be obtained for electronic densities $0 \leqslant n$ $\leqslant 1$ in the limits $U / t \rightarrow 0$ and $U / t \gg 1$, with the results

$$
\begin{gathered}
f(n, U / t)=1 ; \quad U / t \rightarrow 0, \\
f(n, U / t)=\left(\frac{4 t}{U}\right)^{2} \ln 2\left[1-\frac{\sin (2 \pi n)}{2 \pi n}\right] ; \quad U / t \gg 1 .
\end{gathered}
$$


For the specific case of half filling, $n=1$, one finds from the use of Eqs. (26) and (35) the following closed-form expression for the function $f(n, U / t),{ }^{35}$

$$
f(1, U / t)=4 \int_{0}^{\infty} d x \frac{J_{0}(x) J_{1}(x)}{1+\cosh \left(\frac{x U}{2 t}\right)} .
$$

According to the general expression (22), the ground-state kinetic energy $T_{0}$ is given by $T_{0}=t \partial E_{G S H} / \partial t,{ }^{34}$ where $E_{G S H}$ stands again for the ground-state energy associated with the Hamiltonian (3).

\section{The electron double-occupation functional}

Our goal is the study of the deviations $\Delta D$ from the ground-state electron double occupation $D_{0}$ given in Eq. (26), generated by changes in the numbers of pseudoparticles, $-1 / 2$ Yang holons, and $-1 / 2 \mathrm{HL}$ spinons associated with transitions to other energy eigenstates. Such a study provides information on the $U / t$ dependence of the electron site distribution configurations of the real-space lattice which describe the energy eigenstates. The above-mentioned deviations are interesting quantities that provide useful information about the localization/delocalization degree of the corresponding elementary quantum objects. Fortunately, the electron double occupation $D$ of the final energy eigenstates can be obtained by means of the general expression (21). Thus one can compute the electron double-occupation deviations $\Delta D \equiv\left[D-D_{0}\right]$. These can be obtained simply by taking the $U$ derivative of the excitation energy defined by the following Landau-liquid energy functional,

$$
\Delta E_{H}=\Delta E-\sum_{\alpha=c, s} \mu_{\alpha} \Delta S_{z}^{\alpha}-\frac{U}{2}\left[\Delta L_{c,+1 / 2}-\Delta L_{c,-1 / 2}\right],
$$

where $\Delta E$ is the energy functional (24), $\Delta L_{c, \pm 1 / 2}$ denotes the deviations in the numbers of $\pm 1 / 2$ Yang holons, and the remaining quantities are the same as on the right-hand side of Eq. (20).

The deviations $\Delta D$ can be written in functional form. To first order in the $-1 / 2$ Yang holon and $-1 / 2$ HL spinon number deviations and pseudoparticle band-momentum distribution function deviations, the double-occupation functional reads

$$
\begin{aligned}
\Delta D= & \sum_{\alpha=c, s} \Delta L_{\alpha,-1 / 2} D_{\alpha,-1 / 2}+\frac{N_{a}}{2 \pi} \int_{q_{c}^{-}}^{q_{c}^{+}} d q \Delta N_{c}(q) D_{c}(q) \\
& +\frac{N_{a}}{2 \pi} \sum_{\alpha=c, s} \sum_{\nu=1}^{\infty} \int_{-q_{\alpha, \nu}}^{q_{\alpha, \nu}} d q \Delta N_{\alpha, \nu}(q) D_{\alpha, \nu}(q) .
\end{aligned}
$$

Here $\Delta L_{\alpha,-1 / 2}$ is the deviation from the ground-state number of $-1 / 2$ Yang holons $(\alpha=c)$ and $-1 / 2$ HL spinons ( $\alpha$ $=s)$, and $\Delta N_{c}(q)$ and $\Delta N_{\alpha, \nu}(q)$ are the $c$ - and $\alpha, \nu$-pseudoparticle band-momentum distribution function deviations given in Eq. (58) of Ref. 31, respectively. On the right-hand side of Eq. (39) $D_{\alpha,-1 / 2}$ is the $-1 / 2$ Yang holon $(\alpha=c)$ and $-1 / 2$ HL spinon $(\alpha=s)$ double occupation, while $D_{c}(q)$ and $D_{\alpha, \nu}(q)$ denote the $c$-pseudoparticle and $\alpha, \nu$-pseudoparticle double-occupation spectra, respectively. These double occupations and spectra equal the corresponding deviation in the value of double occupation (18) which results from creation of a $-1 / 2$ Yang holon or $-1 / 2 \mathrm{HL}$ spinon and of creation of a $c$ or $\alpha, \nu$ pseudoparticle at band momentum $q$, respectively. The pseudoparticle doubleoccupation spectra can be expressed as

$$
D_{c}(q)=\frac{\partial \overline{\boldsymbol{\epsilon}}_{c}(q)}{\partial U}, \quad D_{\alpha, \nu}(q)=\frac{\partial \overline{\boldsymbol{\epsilon}}_{\alpha, \nu}(q)}{\partial U},
$$

where the pseudoparticle bands $\bar{\epsilon}_{c}(q)$ and $\bar{\epsilon}_{\alpha, \nu}(q)$ are given by

$$
\begin{gathered}
\bar{\epsilon}_{c}(q)=\epsilon_{c}(q)+U / 2-\mu+\mu_{0} H, \quad \bar{\epsilon}_{s, 1}(q)=\epsilon_{s, 1}(q)-2 \mu_{0} H, \\
\bar{\epsilon}_{c, \nu}(q)=\epsilon_{c, \nu}^{0}(q)+\nu U, \quad \bar{\epsilon}_{s, \nu}=\epsilon_{s, \nu}^{0}(q) ; \quad \nu>1 .
\end{gathered}
$$

Here the pseudoparticle energy bands $\epsilon_{c}(q), \boldsymbol{\epsilon}_{s, 1}(q)$, $\epsilon_{c, \nu}^{0}(q)$, and $\epsilon_{s, \nu}^{0}(q)$ for $\nu>1$ are defined by Eqs. (C15)(C21) of Ref. 31. These are the energy bands of the energy functional (24). The $-1 / 2$ Yang holon and $-1 / 2 \mathrm{HL}$ spinon electron double-occupation deviation numbers are simply given by

$$
D_{c,-1 / 2}=1 ; \quad D_{s,-1 / 2}=0 .
$$

As for electron double occupation, we could also introduce a kinetic-energy functional associated with the kineticenergy deviations $\Delta T \equiv\left[T-T_{0}\right]$. However, the only information we need for our present study is whether creation of an elementary quantum object leads to a finite or a vanishing kinetic-energy deviation $\Delta T$.

The pseudoparticle electron double-occupation spectra (40) and $-1 / 2$ Yang holon and $-1 / 2$ HL spinon electron double occupation (42) provide interesting physical information about the degree of localization/delocalization of the corresponding quantum objects. For instance, Eq. (42) reveals that for the whole parameter space creation of a $-1 / 2$ Yang holon leads to $\Delta D=1$. That excitation involves creation of two electrons. From the expression of the $\eta$-spin-flip generators given in Eq. (9), it follows that creation of a $-1 / 2$ Yang holon involves the creation of an extra on-site electron pair on a ground-state empty site for all realspace lattice electron site distribution configurations which describe the initial state. Consistently, we find that creation of a $-1 / 2$ Yang holon leads to a kinetic-energy deviation $\Delta T=0$. Since creation of such quantum objects leads to no change in the value of the ground-state kinetic energy, we say that the $-1 / 2$ Yang holon carries no kinetic energy. Therefore, in spite of having charge $-2 e,-1 / 2$ Yang holons do not contribute directly to charge transport, as mentioned in Ref. 31. This concept of an elementary quantum object carrying kinetic energy when its creation leads to a finite deviation $\Delta T$ is inspired in the electronic conductivity sum rule. That sum rule refers to the frequency-dependent electronic conductivity and states that it is proportional to $|T|{ }^{34}$ Thus if creation of a quantum object does not change the 
value of $T$, it follows that the conductivity sum rule remains unchanged. Therefore, we say that such a quantum object carries no kinetic energy. Furthermore, we find from analysis of Eq. (42) that creation of a $-1 / 2 \mathrm{HL}$ spinon leads to no change in the ground-state double occupation. This is consistent with the form of the generators defined in Eq. (10). Thus the spin flip associated with creation of a $-1 / 2 \mathrm{HL}$ spinon corresponds to electrons located at singly occupied sites for all real-space lattice electron site distribution configurations which describe the initial ground state.

Let us start the discussion of the pseudoparticle electron double-occupation spectra defined in Eq. (40) by considering the limit $U / t \rightarrow \infty$. These spectra can be easily evaluated and in that limit the elementary quantum objects have simpler expressions in terms of real-space lattice electron site distribution configurations. As discussed in Refs. 31 and 32, such $U / t \rightarrow \infty$ configurations describe the energy eigenstates for all values of $U / t$ in terms of an effective electronic lattice. Such a lattice refers to the occupancy configurations of the rotated electrons introduced in Ref. 32. It has the same number of sites $N_{a}$ and lattice constant $a$ as the real-space lattice. ${ }^{31,32}$ In the limit of $U / t \rightarrow \infty$, electron double occupation $D$ is a good quantum number which equals the number of rotated-electron doubly occupied sites. ${ }^{31,32}$ We recall that for rotated electrons double occupation is a good quantum number for all values of $U / t$. As a result we find that as $U / t \rightarrow \infty$ the electron double-occupation functional (39) simply reads

$$
\begin{aligned}
\Delta D= & \Delta M_{c,-1 / 2}=\Delta L_{c,-1 / 2} \\
& +\frac{N_{a}}{2 \pi} \sum_{\nu=1}^{\infty} \int_{-q_{c, \nu}}^{q_{c, \nu}} d q \nu \Delta N_{c, \nu}(q) ; \quad U / t \rightarrow \infty .
\end{aligned}
$$

From comparison of Eqs. (39) and (43), we arrive at the following $U / t \rightarrow \infty$ expressions for the pseudoparticle electron double-occupation spectra,

$$
D_{c}(q)=D_{s, \nu}(q)=0 ; \quad D_{c, \nu}(q)=\nu ; \quad U / t \rightarrow \infty .
$$

These electron double-occupation spectra expressions show that increasing the number of $c$ pseudoparticles by one does not change electron double occupation when $U / t \rightarrow \infty$. On the other hand, from Eq. (44) we find that increasing the number of $c, \nu$ pseudoparticles by one leads to an increase $\nu$ in electron double occupation $D$. Moreover, we also studied the corresponding deviations in kinetic energy and found that in this limit the $c$ pseudoparticles ( $c, \nu$ pseudoparticles) carry kinetic energy (carry no kinetic energy).

These results reveal that in the present limit, the $c$ pseudoparticles correspond to electron singly occupied sites and that the $-1 / 2$ holons and $+1 / 2$ holons correspond to electron pairs and electronic hole pairs at the same real-space lattice site, respectively. In Ref. 32 it is found that such a property is closely related to the rotated-electron effective electronic lattice mentioned above. As we discuss below, we find that at finite values of $U / t$ a composite $2 \nu$-holon $c, \nu$ pseudoparticle with band momentum $q$ such that $|q|<[\pi$ $\left.-2 k_{F}\right]$ carries kinetic energy. This means that for finite values of $U / t$ the $2 \nu$ extra electrons are not always created at the same site and thus that composite quantum object contributes to charge transport. However, we find that $\Delta T$ is a decreasing function of $U / t$ which vanishes in the limit $U / t$ $\rightarrow \infty$. Thus creation of a $c, \nu$ pseudoparticle leads in that limit to $\Delta T=0$ and these objects become localized and carry no kinetic energy. It follows that in spite of having charge $-2 \nu e$, for $U / t \rightarrow \infty$ the $c, \nu$ pseudoparticles do not contribute directly to charge transport, as mentioned in Ref. 31. In that limit they describe a number $\nu$ of localized electron pairs, each pair localized at the same site.

On the other hand, in agreement with the results of Ref. 31 the chargeons have charge $-e$ and we find that in the $U / t \rightarrow \infty$ limit they carry kinetic energy and thus are the only carriers of charge. When $U / t \rightarrow \infty$ the finite-energy Hilbert subspace is spanned by states with $D=0$ because it requires an infinite energy, $D U$, to excite the system into states with $D>0$ electron double occupation. In this limit, the $c$ pseudoparticles become the spinless fermions of Refs. 5457. The associated chargeons describe the charge excitations associated with the electron singly occupied sites of the realspace lattice. The spin degrees of freedom of the electron singly occupied sites are described by the spinons. In the $U / t \rightarrow \infty$ limit creation of $\pm 1 / 2$ spinons describes spin flips of localized electrons at singly occupied sites. Therefore, creation of spinons does not contribute to double occupation, as confirmed by the results of Eq. (43) for the $2 \nu$-spinon composite $s, \nu$ pseudoparticles. This holds true for all finite values of $U / t$ in the case of the HL spinons, as confirmed by Eq. (42). Moreover, creation of single $\pm \frac{1}{2}$ spinons associated with creation of single electrons describes creation of localized spin $\pm \frac{1}{2}$ electrons singly occupied sites and annhilation of empty sites.

\section{Electron double-occupation spectra for finite values of $U / t$ and zero-spin density}

Let us now study the pseudoparticle electron doubleoccupation spectra of Eq. (40) for finite values of $U / t$. For simplicity, let us again consider the case of zero-spin density $m=0$. In that case we find the following relations, which are valid for values of electronic density in the domain $0 \leqslant n$ $\leqslant 1$ and for all values of $U / t$,

$$
\begin{gathered}
D_{s, \nu}(q)=0 ; \quad \nu>1, \\
D_{c, \nu}(0) \leqslant D_{c, \nu}(q) \leqslant D_{c, \nu}\left( \pm\left[\pi-2 k_{F}\right]\right)=\nu ; \\
D_{s, 1}\left( \pm k_{F}\right)=0 \leqslant D_{s, 1}(q) \leqslant D_{s, 1}(0) .
\end{gathered}
$$

The electron double-occupation spectra are derived from the expressions of Eq. (41) for the energy bands $\bar{\epsilon}_{c}(q)$ and $\bar{\epsilon}_{c, \nu}(q)$ by means of the relation provided in Eq. (40). Therefore, the features of these bands fully control and determine the band momentum $q$, electronic density $n$, and on-site repulsion $U / t$ dependence of the pseudoparticle electron double-occupation spectra. As we discuss in the ensuing section, the energy width of the band $\bar{\epsilon}_{c}(q)$ is independent of 
$U / t$, being constant for all values of $U / t$ and $n$, and is given by $W_{c} \equiv\left|\bar{\epsilon}_{c}(\pi)-\bar{\epsilon}_{c}(0)\right|=4 t$. In contrast, the width $W_{c, \nu}$ $\equiv\left|\bar{\epsilon}_{c, \nu}\left(\pi-2 k_{F}\right)-\bar{\epsilon}_{c, \nu}(0)\right|$ of the $c$-, $\nu$-pseudoparticle energy bands is a decreasing function of $U / t$, vanishing as $U / t \rightarrow \infty$. The same behavior occurs also for the $s, 1$-pseudoparticle band, which is such that $W_{s, 1} \equiv \mid \bar{\epsilon}_{s, 1}\left(k_{F}\right)$ $-\bar{\epsilon}_{s, 1}(0) \mid \rightarrow 0$ as $U / t \rightarrow \infty$.

This qualitative difference between the $c$-pseudoparticle and the remaining pseudoparticle energy bands leads to qualitative differences in the corresponding electron doubleoccupation spectra as well. For instance, there are qualitative differences between the $q$ dependence of the electron doubleoccupation spectrum $D_{c}(q)$ on the one hand, and that of the electron double-occupation spectra $D_{s, 1}(q)$ and $D_{c, \nu}(q)$ on the other hand. While the function $D_{c}(q)$ changes slowly with band momentum $q$, for finite values of $U / t$ the functions $D_{s, 1}(q)$ and $D_{c, \nu}(q)$ show a significant $q$ dependence. For instance, the values $D_{s, 1}\left(k_{F}\right)=0$ and $D_{c, \nu}\left(\pi-2 k_{F}\right)=\nu$ are $U / t$ independent, whereas the values of $D_{s, 1}(0)$ and $D_{c, \nu}(0)$ depend on this quantity. In contrast, the $U / t$ dependence of the spectrum $D_{c}(q)$ is very similar for all band-momentum values $q$. For finite values of $U / t$ the electron doubleoccupation spectrum $D_{s, 1}(q)=D_{s, 1}(-q)$ is a decreasing function of $|q|$, having its minimum value $D_{s, 1}\left(k_{F}\right)=0$ at $|q|=q_{s, 1}=k_{F}$ and its maximum value at $|q|=0$. For finite values of $U / t$ the electron double-occupation spectrum $D_{c, \nu}(q)=D_{c, \nu}(-q)$ is an increasing function of $|q|$, having its minimum value at $|q|=0$ and its maximum value $D_{c, \nu}\left(\pi-2 k_{F}\right)=\nu$ at $|q|=q_{c, \nu}=\left[\pi-2 k_{F}\right]$.

Since both the electron double-occupation spectra $D_{s, 1}(q)$ and $D_{c, \nu}(q)$ are not dependent on $U / t$ for the limiting bandmomentum values $q= \pm k_{F}$ and $q= \pm\left[\pi-2 k_{F}\right]$, respectively, and they show the strongest $U / t$ dependence for $q$ $=0$, below we consider mainly the $U / t$ and $n$ dependencies of the $q=0$ parameters $D_{s, 1}(0)$ and $D_{c, \nu}(0)$. In agreement with the inequalities of Eq. (46), for increasing values of the band-momentum absolute value $|q|$, these spectra reach smoothly the $U / t$-independent limiting values $D_{s, 1}\left( \pm k_{F}\right)$ $=0$ and $D_{c, \nu}\left( \pm\left[\pi-2 k_{F}\right]\right)=\nu$, respectively. According to the results obtained in Ref. 32, at $m=0$ only the $c$-pseudoparticle; $s, 1$-pseudoparticle; $c, 1$-pseudoparticle; and $c, 2$-pseudoparticle bands contribute significantly to the energy spectra of one-electron and two-electron excitations. Thus, below we limit our studies of the spectrum $D_{c, \nu}(0)$ to the branches $\nu=1$ and $\nu=2$. In the case of the $D_{c}(q)$ spectrum, we consider the $n$ and $U / t$ dependencies of both the parameters $D_{c}(0)$ and $D_{c}\left(-2 k_{F}\right)=D_{c}\left(2 k_{F}\right)$. Although the spectrum $D_{c}(q)$ is gently dependent on $q$, in addition to the value at the band momentum $q=0$ we also consider the value at $\pm 2 k_{F}$. On the one hand, we study the parameter $D_{c}\left(2 k_{F}\right)$ because creation and annihilation of $c$ pseudoparticles at the Fermi points $\pm 2 k_{F}$ are associated with important one-electron and two-electron excitations. ${ }^{47}$ On the other hand, since for the other pseudoparticle branches we study the $U / t$ and $n$ dependencies of the electron doubleoccupation spectrum at $q=0$, for completeness we also consider in our study the parameter $D_{c}(0)$.

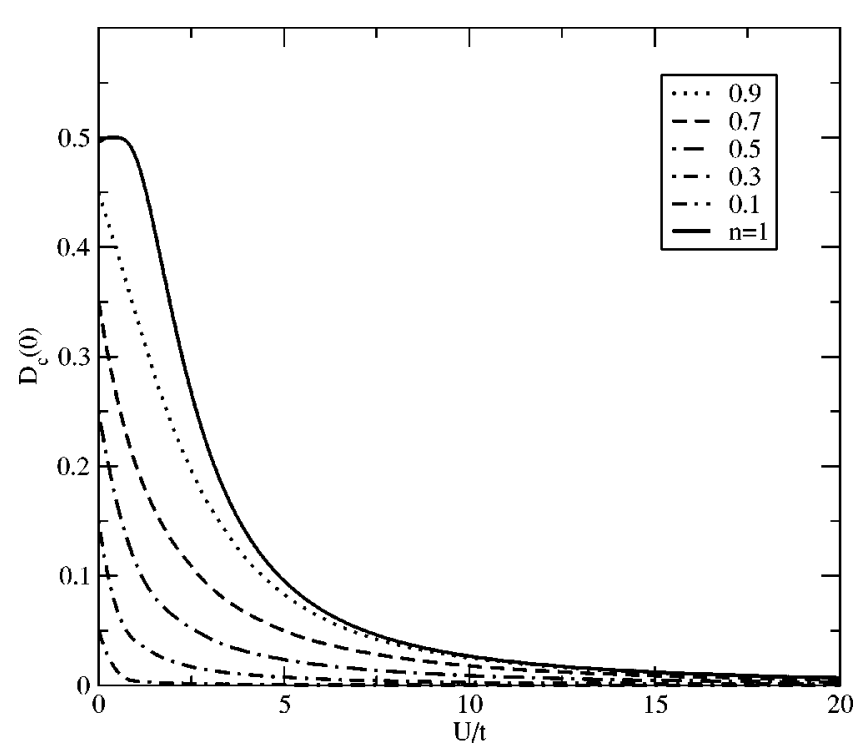

FIG. 1. The electron double-occupation parameter $D_{c}(0)$ plotted as a function of $U / t$ for different values of the electronic density $n$.

In Figs. 1 and 2 the electron double-occupation pseudoparticle parameters $D_{c}(0)$ and $D_{c}\left(2 k_{F}\right)$ are plotted as a function of $U / t$ for different values of the electronic density $n$. The electron double-occupation pseudoparticle parameters $D_{s, 1}(0), D_{c, 1}(0)$, and $D_{c, 2}(0)$ are also plotted as a function of $U / t$ and for the same values of the electronic density in Figs. 3-5. The curves plotted in these figures show that $D_{c}(0)$ and $D_{c}\left(2 k_{F}\right)$ are decreasing functions of $U / t$. Both of these functions have the maximum value $D_{c}(0)=D_{c}\left(2 k_{F}\right)=n / 2$, for $U / t \rightarrow 0$, and vanish in the limit $U / t \rightarrow \infty$. On the other hand, $D_{s, 1}(0)$ vanishes both for $U / t$ $\rightarrow 0$ and $U / t \rightarrow \infty$ and has its $n$-dependent maximum value for a value of $U / t$ between 1 and 4 . While for small values

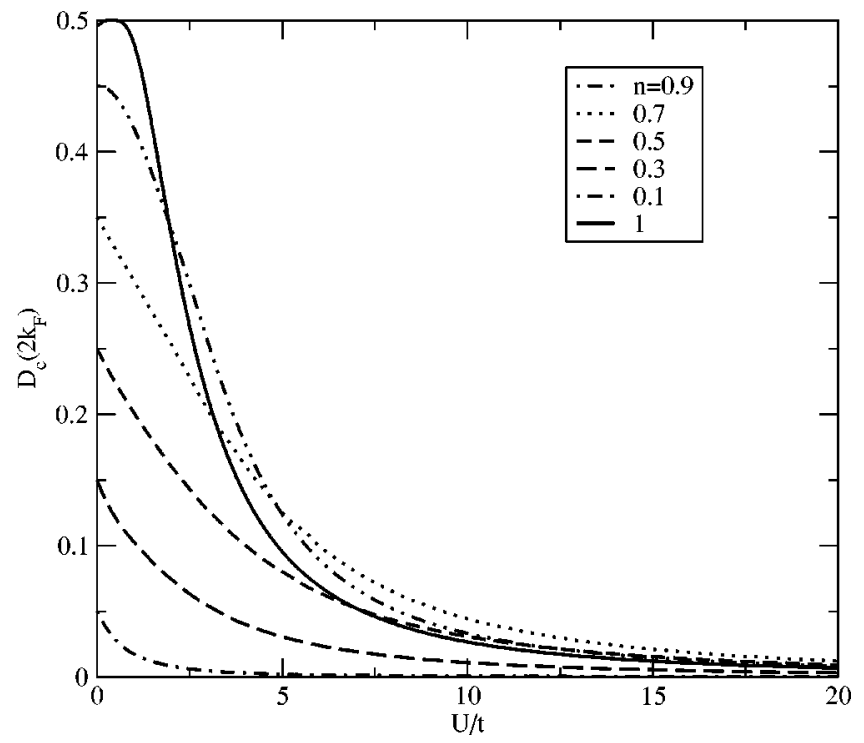

FIG. 2. The electron double-occupation parameter $D_{c}\left(2 k_{F}\right)$ plotted as a function of $U / t$ for different values of the electronic density $n$. 


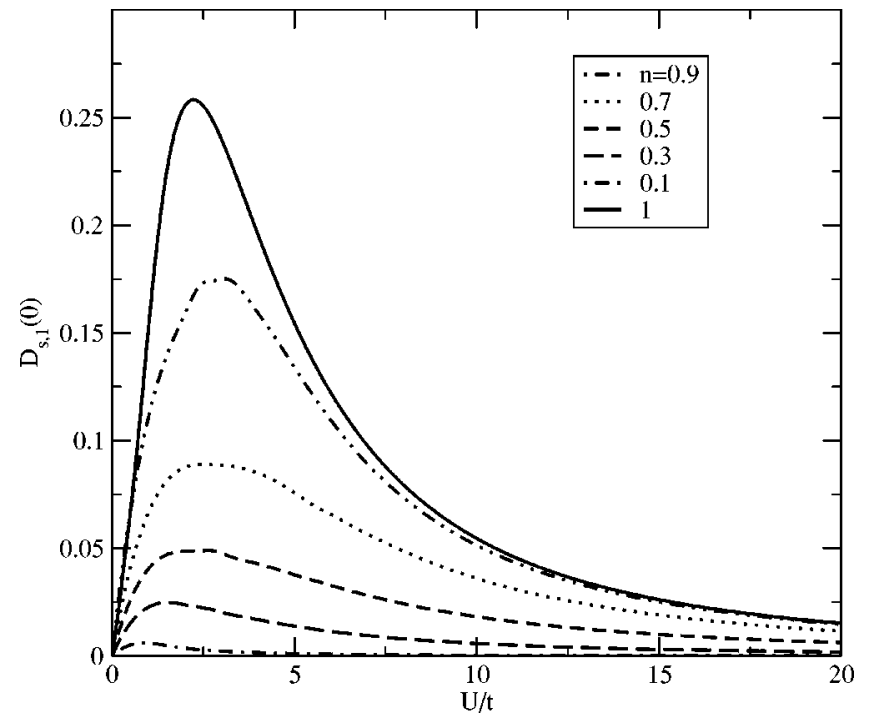

FIG. 3. The electron double-occupation parameter $D_{s, 1}(0)$ plotted as a function of $U / t$ for different values of the electronic density $n$.

of the electronic density $n$ the parameters $D_{c, 1}(0)$ and $D_{c, 2}(0)$ are decreasing functions of $U / t$, for values of $n$ close to 1 there is a minimum value at a small but finite value of $U / t$. These parameters are given by $n$ and $2 n$, respectively, for $U / t \rightarrow 0$, and tend to 1 and 2 , respectively, as $U / t \rightarrow \infty$.

Before discussing the physical information contained in the curves plotted in Figs. 1-5, we consider some limiting cases where we could evaluate analytical expressions for the pseudoparticle electron double-occupation spectra. In addition, before that discussion we also indicate the cases in which creation of the elementary quantum objects leads to a vanishing kinetic-energy deviation $\Delta T$. In the limit $U / t \rightarrow 0$

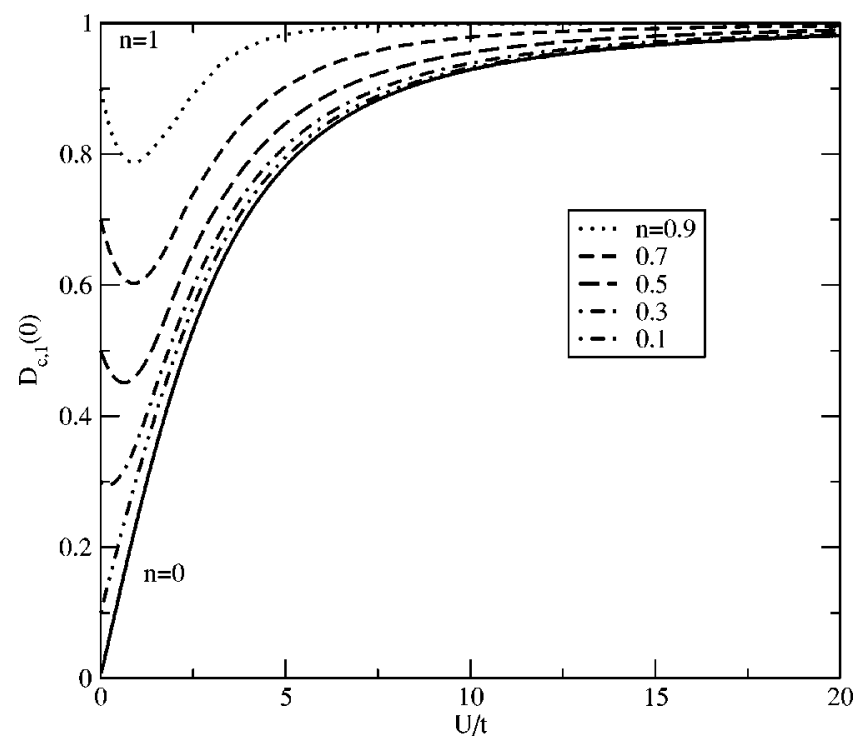

FIG. 4. The electron double-occupation parameter $D_{c, 1}(0)$ plotted as a function of $U / t$ for different values of the electronic density $n$. we find the following expressions valid for densities $0 \leqslant n$ $\leqslant 1$

$$
D_{c}(0)=D_{c}\left( \pm 2 k_{F}\right)=n / 2 ; \quad D_{s, 1}(0)=0 ; \quad D_{c, \nu}(0)=\nu n \text {. }
$$

On the other hand, in the limit $U / t \gg 1$ the same quantities can be written as follows,

$$
\begin{gathered}
D_{c}(0)=\left(\frac{2 t}{U}\right)^{2} n \ln (2)\left[1-\frac{\sin (2 \pi n)}{2 \pi n}\right] \\
D_{c}\left( \pm 2 k_{F}\right)=\left(\frac{2 t}{U}\right)^{2} n \ln (2)\left[2 \sin ^{2}(\pi n)+1-\frac{\sin (2 \pi n)}{2 \pi n}\right] ; \\
D_{s, 1}(0)=\left(\frac{2 t}{U}\right)^{2} \frac{\pi n}{2}\left[1-\frac{\sin (2 \pi n)}{2 \pi n}\right] ; \\
D_{c, \nu}(0)=\nu-\left(\frac{4 t}{U}\right)^{2} \frac{1}{2 \nu}\left\{(1-n)-\frac{\sin [2 \pi(1-n)]}{2 \pi}\right\} .
\end{gathered}
$$

Moreover, the following expressions are valid for all values of $U / t>0$ in the limit of vanishing density $n \rightarrow 0$,

$$
\begin{gathered}
D_{c}(0)=D_{c}\left( \pm 2 k_{F}\right)=D_{s, 1}(0)=0 ; \\
D_{c, \nu}(0)=\nu \frac{\nu U}{\sqrt{(4 t)^{2}+(\nu U)^{2}}} .
\end{gathered}
$$

In the case of density $n=1$ we could derive the following expressions, which are valid for all values of $U / t>0$,

$$
\begin{gathered}
D_{c}(0)=D_{c}\left( \pm 2 k_{F}\right)=\int_{0}^{\infty} d x \frac{J_{1}(x)}{1+\cosh \left(\frac{x U}{2 t}\right)} \\
D_{s, 1}(0)=\frac{1}{2} \int_{0}^{\infty} d x J_{1}(x) \frac{\sinh \left(\frac{x U}{4 t}\right)}{\left[\cosh \left(\frac{x U}{4 t}\right)\right]^{2}} ; \quad D_{c, \nu}(0)=\nu .
\end{gathered}
$$

Comparison of the expressions (47)-(50) with the curves of the Figs. 1-5 reveals there is agreement between these expressions and the curves.

As we mentioned above, we can also evaluate the kineticenergy deviations $\Delta T \equiv\left[T-T_{0}\right]$ associated with elementary excitations. In the present study we are mostly interested in whether the deviations $\Delta T$ which result from creation of a pseudoparticle, $-1 / 2$ Yang holon, or $-1 / 2$ HL spinon are finite or vanish. Importantly, by combining our analysis of the $\Delta T$ deviation expression with the related properties of the electron double-occupation spectra, we find that when such a deviation vanishes, creation of these quantum objects is either associated with creation of localized electrons or involves on-site spin-flip processes. From our study of the kinetic energy (19) we find that the deviation $\Delta T$ which re- 


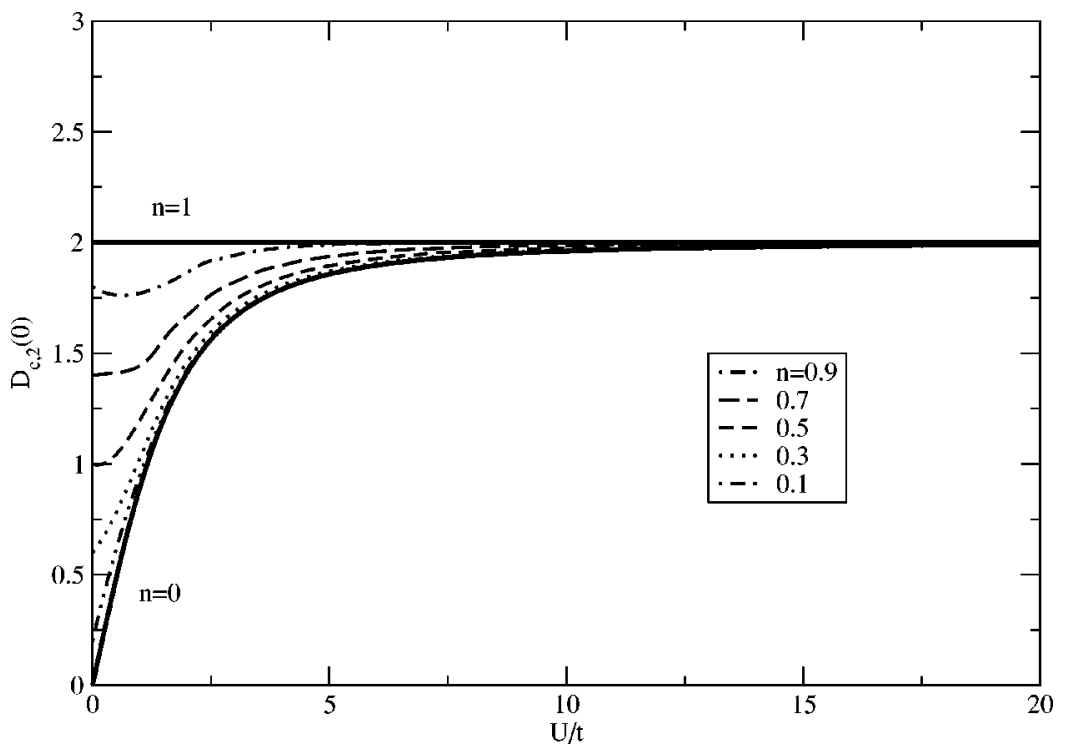

FIG. 5. The electron double-occupation parameter $D_{c, 2}(0)$ plotted as a function of $U / t$ for different values of the electronic density $n$. sults from creation of pseudoparticles, - 1/2 Yang holons, or $-1 / 2$ HL spinons vanishes in the following cases:

(i) Creation of one or a vanishing density of $s, 1$ pseudoparticles at band-momentum values $q= \pm k_{F \uparrow}$ for all values of $U / t, n$, and $m$.

(ii) Creation of one or a vanishing density of $c, \nu$ pseudoparticles at band-momentum values $q= \pm\left[\pi-2 k_{F}\right]$ for all values of $U / t, n$, and $m$.

(iii) Creation of one or a vanishing density of $s, \nu$ pseudoparticles belonging to branches such that $\nu>1$ at band-momentum values $q= \pm\left[k_{F \uparrow}-k_{F \downarrow}\right]$ for all values of $U / t, n$, and $m$.

(iv) Creation of $-1 / 2$ Yang holons for all values of $U / t$, $n$, and $m$.

(v) Creation of $-1 / 2 \mathrm{HL}$ spinons for all values of $U / t, n$, and $m$.

(vi) Creation of $\alpha, \nu$ pseudoparticles with $\alpha=c, s$ and $\nu$ $=1,2,3, \ldots$ for $m=0$, all band-momentum values $q$, all densities $n$, and $U / t \rightarrow \infty$.

\section{E. Electron double-occupation spectra and the pseudoparticle degree of localization/delocalization}

We close this section with the analysis and a discussion of the information provided by the dependence on the electronic density $n$, on-site electronic repulsion $U / t$, and band momentum $q$ of the electron double-occupation quantities defined in Eqs. (46)-(50) and Figs. 1-5. We find that the dependence of the electron double-occupation quantities and spectra on these parameters provides insight into the degree of localization/delocalization of the $-1 / 2$ Yang holons, $-1 / 2$ HL spinons, and pseudoparticles.

While only spin $\sigma$ electrons can be created into the manyelectron system or annihilated from it, creation of spin $\sigma$ electrons generates backflow effects which can change the numbers and the occupancy configurations of pseudoparticles, holons, and spinons. ${ }^{32}$ In the absence of electron addition or removal, these elementary quantum objects are confined within the many-electron system, their occupancy configurations describing the exact energy eigenstates. As a result of the nonperturbative character of the electronic correlations, the description of the pseudoparticles, holons, and spinons in terms of electron site distribution configurations of the real-space lattice is a very complex many-body problem. $^{31}$

For finite values of $U / t$ the electron double occupation $D$ defined in Eq. (18) is not a good quantum number. This just means that the ground state and the other energy eigenstates can be expressed as a superposition of real-space lattice electron site distribution configurations with different numbers of electron doubly occupied sites. On the other hand, in the limit $U / t \rightarrow \infty$ both electron double occupation $D$, double unoccupation, spin-down single occupation, and spin-up single occupation become good quantum numbers. ${ }^{31,32}$ Thus in this limit all energy eigenstates can be expressed as a superposition of real-space lattice electron site distribution configurations with the same value of electron double occupation $D$. For instance, in the particular case of the ground state and finite-energy excited states that value is $D=0$. Thus, the ground-state electron double occupation $D_{0}$ is such that $D_{0}$ $\rightarrow 0$ as $U / t \rightarrow \infty$, as confirmed by the form of the expression defined in Eqs. (26) and (36).

It is useful for our discussion to express the deviations $\Delta N_{\uparrow}$ and $\Delta N_{\downarrow}$ in the electron numbers in terms of the deviations in the $c$ pseudoparticle; $\alpha, \nu$ pseudoparticle, $-1 / 2$ Yang holon; and $-1 / 2 \mathrm{HL}$ spinon numbers. Based on the form of the expressions given in Eqs. (14)-(16), we find the following relations,

$$
\begin{aligned}
\Delta N_{\uparrow}= & \Delta N_{c}-\Delta L_{s,-1 / 2}+\Delta L_{c,-1 / 2} \\
& +\sum_{\nu=1}^{\infty} \nu \Delta N_{c, \nu}-\sum_{\nu=1}^{\infty} \nu \Delta N_{s, \nu},
\end{aligned}
$$




$$
\Delta N_{\downarrow}=\Delta L_{s,-1 / 2}+\Delta L_{c,-1 / 2}+\sum_{\nu=1}^{\infty} \nu \Delta N_{c, \nu}+\sum_{\nu=1}^{\infty} \nu \Delta N_{s, \nu} .
$$

We recall that the numbers of $+1 / 2$ Yang holons and of $+1 / 2$ HL spinons are dependent on the numbers of the remaining quantum objects. In particular, it follows from Eq. (17) that the deviations $\Delta L_{c,+1 / 2}$ and $\Delta L_{s,+1 / 2}$ can be expressed as follows,

$$
\begin{gathered}
\Delta L_{c,+1 / 2}=-\Delta N_{c}-2 \sum_{\nu=1}^{\infty} \nu \Delta N_{c, \nu}-\Delta L_{c,-1 / 2} \\
\Delta L_{s,+1 / 2}=\Delta N_{c}-2 \sum_{\nu=1}^{\infty} \nu \Delta N_{s, \nu}-\Delta L_{s,-1 / 2} .
\end{gathered}
$$

For simplicity, let us assume again that the initial ground state has zero-spin density $m=0$. In order to study the degree of localization/delocalization of the quantum objects, we consider elementary excitations associated with creation of $c$ pseudoparticles; $s, 1$ pseudoparticles; $c, \nu$ pseudoparticles; and $-1 / 2$ Yang holons. According to Eqs. (24) and (25), for initial ground states with finite spin density $m$ in the range $0<m<n$ the energy spectrum of excitations involving creation of $s, \nu$ pseudoparticles belonging to $\nu>1$ branches and/or $-1 / 2$ Yang holons is gapped. The study of the electron double occupation of such finite-spin-density excitations is an interesting problem which will be considered elsewhere.

In the examples provided in the following we consider the simplest excitations associated with creation of one pseudoparticle at band-momentum $q$ (or of one $-1 / 2$ Yang holon or $-1 / 2 \mathrm{HL}$ spinon). However, note that the contribution to the electron double-occupation deviation $\Delta D$ of any other excitations involving creation of the same quantum object always is given by its double occupation spectrum at band-momentum $q$ (or $-1 / 2$ Yang holon or $-1 / 2$ HL spinon double occupation).

We start by considering the creation of a $c$ pseudoparticle at band momentum $q$. Creation of a $c$ pseudoparticle is associated with creation of a spin-up electron, as confirmed by Eqs. (51) and (52). From Eq. (53) we find that such an excitation also involves annihilation of a $+1 / 2$ Yang holon and creation of a $+1 / 2 \mathrm{HL}$ spinon. According to Eqs. (43) and (48), in the limit $U / t \rightarrow \infty$ this elementary excitation leads to no change in the ground-state electron double occupation $D_{0}$, Eq. (26). Therefore, in this limit creation of a $c$ pseudoparticle corresponds to creation of an extra electron singly occupied site and annihilation of an empty side of the real-space lattice. For decreasing values of $U / t$, the $U / t$ dependence of the $c$-pseudoparticle electron double occupation of Figs. 1 and 2 reveals that this excitation leads to a small positive finite electron double-occupation deviation $\Delta D$ $=D_{c}(q)$. At both $q=0$ and $q= \pm 2 k_{F}$ this deviation is maximum when $U / t \rightarrow 0$, where from Eq. (47) and Figs. 1 and 2 we find that $\Delta D=D_{c}(0)=D_{c}\left( \pm 2 k_{F}\right)=n / 2$. The deviation $\Delta D=D_{c}(q)$ changes slowly with band momentum $q$ for all values of $U / t$ and electronic density $n$. We emphasize that the kinetic-energy deviation $\Delta T$ which results from this excitation is always finite. We thus conclude that the $c$ pseudoparticle does not describe localized electron configurations. The $c$ pseudoparticle has it highest delocalization degree in the limit of $U / t \rightarrow \infty$, where it leads to no electron double-occupation deviation. In this case it becomes the noninteracting spinless fermion of Refs. 54-57.

Let us now consider the case of the $s, 1$ pseudoparticle. Creation of an $s, 1$ pseudoparticle at band momentum $q$ is associated with a spin flip which creates a spin-down electron and annihilates a spin-up electron. This is confirmed by analysis of Eqs. (51) and (52). Moreover, it follows from Eq. (53) that creation of an $s, 1$ pseudoparticle also involves annihilation of two $+1 / 2$ HL spinons. The corresponding electron double-occupation deviation is given by $\Delta D=D_{s, 1}(q)$ and equals the $s, 1$ double-occupation spectrum. According to Eqs. (46)-(48), this spectrum has its minimum value at the band-momentum values $q= \pm k_{F}$, where it vanishes. In addition, we find that at this band-momentum value the $s, 1$ pseudoparticle does not carry kinetic energy. Thus at $q$ $= \pm k_{F}$ and for all finite values of $U / t$ and $n$, creation of the $s, 1$ pseudoparticle is associated with an on-site electronic spin-flip process in the real-space lattice. It follows that at these band-momentum values the $s, 1$ pseudoparticle has a localized character. For decreasing values of $|q|$ and finite values of $U / t$, the value of $D_{s, 1}(q)$ increases and we find that the $s, 1$ pseudoparticle carries kinetic energy. It follows that for $|q|<k_{F}$, the spin-flip electronic process has no pure on-site character. The delocalization degree of the $s, 1$ pseudoparticle is maximum for band momentum $q=0$. In contrast to the $c$ pseudoparticles and $c, \nu$ pseudoparticles, at finite values of $U / t$, the degree of delocalization of the $s, 1$ pseudoparticle is in general highest when the value of $D_{s, 1}(q)$ is maximum. This can be understood by considering the $U / t \rightarrow \infty$ limit. In this case the $q= \pm k_{F}$ physics is extended to all values of $q$ and the $s, 1$ pseudoparticle carries no kinetic energy. Moreover, according to Eqs. (43) and (48) the electron double-occupation spectrum is in this limit given by $D_{s, 1}(q)=0$. As was mentioned before, creation of the $s, 1$ pseudoparticle at any value of $q$ for $U / t \rightarrow \infty$ is associated with an on-site electronic spin-flip process. It follows that in the case of the $s, 1$ pseudoparticle the highest degree of localization is achieved when it describes electron singly occupied site excitations only. Thus, as the value of the on-site repulsion $U / t$ is decreased and $\Delta D=D_{s, 1}(q)$ becomes finite, the degree of delocalization increases. This effect is stronger for low values of band momentum $q$. For the limiting values $q= \pm k_{F}$ the $s, 1$ pseudoparticle describes a localized excitation for all values of $U / t$ and $n$, as mentioned above. The curves plotted in Fig. 3 show that $\Delta D=D_{s, 1}(0)$ is maximum for an intermediate value of $U / t$. Interestingly, in the limit of $U / t \rightarrow 0$ one finds that $D_{s, 1}(q)=0$ for all values of $q$, as in the limit $U / t \rightarrow \infty$. However, in this case the $s, 1$ pseudoparticle carries finite kinetic energy for all band-momentum values $q$ except at $q= \pm k_{F}$. This means that for vanishing values of $U / t$ the delocalization degree is not directly related to electron double occupation. 
Another important elementary quantum object is the $-1 / 2$ Yang holon. Creation of a $-1 / 2$ Yang holon is associated with creation of two electrons of opposite spin projection, as is confirmed by Eqs. (51) and (52). From Eq. (53) we find that creation of $\mathrm{a}-1 / 2$ Yang holon implies annihilation of a $+1 / 2$ Yang holon. The $-1 / 2$ Yang holon does not carry kinetic energy and from Eq. (42) we find that its creation leads to a electron double-occupation deviation value $\Delta D$ $=1$. Therefore, creation of a $-1 / 2$ Yang holon is associated for all finite values of $U / t$ with the creation of a new localized electron doubly occupied site and the annihilation of an empty site in all real-space lattice electron site distribution configurations of the initial ground state, as mentioned previously. This is fully confirmed by the expression in terms of electronic operators of the $\eta$-spin-flip generators given in Eq. (9).

Let us now consider the $c, \nu$ pseudoparticle. This is an $s_{c}=0$ composite quantum object which results from the combination of $\nu-1 / 2$ holons and $\nu+1 / 2$ holons. ${ }^{31}$ Since each $-1 / 2$ holon is made out of two rotated electrons of opposite spin projection, ${ }^{31,32}$ it follows that creation of a $c, \nu$ pseudoparticle at band momentum $q$ is associated with creation of a number $\nu$ of spin-down electrons and creation of an equal number of spin-up electrons. (We note that the number of rotated electrons equals that of electrons.) This is confirmed by Eqs. (51) and (52). According to Eq. (53), creation of a $c, \nu$ pseudoparticle also implies annihilation of $2 \nu+1 / 2$ Yang holons. The electron double-occupation deviation originated by creation of a $c, \nu$ pseudoparticle is given by $\Delta D=D_{c, v}(q)$. According to Eqs. (46)-(48) and Figs. 4 and 5 , this deviation has its maximum value for the bandmomentum values $q= \pm\left[\pi-2 k_{F}\right]$, where it is given by $\nu$. In addition, at these band-momentum values the $c, \nu$ pseudoparticle does not carry kinetic energy. It follows that at these values of $q$, creation of the $c, \nu$ pseudoparticle is associated with creation of $\nu$ localized electron doublyoccupied sites and annihilation of $\nu$ electron empty sites in all real-space lattice electron site distribution configurations of the initial ground state. Note that this result is valid for all electronic densities $n$ and finite values of the on-site repulsion U/t. For these band-momentum values the $c, \nu$ pseudoparticle corresponds to localized real-space lattice electron site distribution configurations similar to the ones obtained by creation of $\nu-1 / 2$ Yang holons. For decreasing values of $|q|$ and finite values of $U / t$ the value $D_{c, \nu}(q)$ decreases and we find that the $c, \nu$ pseudoparticle carries finite kinetic energy. Thus, in contrast to the case of the $s, 1$ pseudoparticle, in the present case the degree of delocalization increases with decreasing electron double-occupation deviation values. For finite values of $U / t$ and bandmomentum values $|q|<\left[\pi-2 k_{F}\right]$ the $2 \nu$ created electrons are characterized by some degree of delocalization which is maximum at $q=0$. This means that for these bandmomentum values the $2 \nu$ electrons have some degree of delocalization and are not all created in pairs at the same site of the real-space lattice. On the other hand, as $U / t \rightarrow \infty$ the $q$ $= \pm\left[\pi-2 k_{F}\right]$ physics is extended to all values of $q$. In this limit the $c, \nu$ pseudoparticle does not carry kinetic energy for all values of $q$ and according to Eq. (48) the electron doubleoccupation spectrum is in that limit given by $D_{c, \nu}(q)=\nu$. As discussed above, in this limit creation of a $c, \nu$ pseudoparticle for all values of $q$ is associated with creation of $\nu$ localized electron double-occupied sites and annihilation of $\nu$ electron empty sites in all real-space lattice electron site distribution configurations of the initial ground state. Finally we emphasize that according to the total-momentum expression (36) of Ref. 31, the $c, \nu$-pseudoparticle momentum spectrum is ( $\pi$ $-q$ ). Thus the band momentum $q=0$ is associated with a contribution to the momentum given by $(\pi-q)=\pi$. This reveals that the maximum degree of delocalization of the $c, \nu$ pseudoparticles corresponds to a finite excitation momentum of $\pi$. Note also that for all the remaining pseudoparticle branches the momentum spectrum equals the band momentum $q$.

\section{F. $U / t \rightarrow \infty$ electron double-occupation selection rules}

We let the operator $\hat{O}_{\mathcal{N}}$ be a product of a finite number,

$$
\mathcal{N}=\sum_{l_{c}, l_{s}= \pm 1} \mathcal{N}_{l_{c}, l_{s}}
$$

of one-electron creation and/or annihilation operators. Here $\mathcal{N} / N_{a}$ is vanishingly small in the present thermodynamic limit, and $\mathcal{N}_{l_{c}, l_{s}}$ is the number of electronic creation and annihilation operators for $l_{c}=-1$ and $l_{c}=+1$, respectively, and with spin down and spin up for $l_{s}=-1$ and $l_{s}=+1$, respectively.

Let us consider states $\hat{O}_{\mathcal{N}}|G S\rangle$ which result from application of a general $\mathcal{N}$-electron operator $\hat{O}_{\mathcal{N}}$ onto a ground state $|G S\rangle$ or onto any eigenstate of the spin $\sigma$ electron numbers. The pseudoparticle, holon, and spinon numbers of the ground state are given in Eqs. (C24) and (C25) of Ref. 31. We consider transformations generated by application of an $\mathcal{N}$-electron operator $\hat{O}_{\mathcal{N}}$ on any eigenstate of the spin $\sigma$ electron numbers and not necessarily on the ground state. We let $|\phi\rangle$ be such a general state. It is not required that such an arbitrary state be an energy eigenstate. The corresponding state $\hat{O}_{\mathcal{N}}|\phi\rangle$ is also an eigenstate of the spin $\sigma=\uparrow, \downarrow$ electron number operators. It belongs to an electron ensemble space whose electron numbers differ from the numbers of the initial state by deviations $\Delta N_{\uparrow}$ and $\Delta N_{\downarrow}$, such that $\Delta N=\Delta N_{\uparrow}$ $+\Delta N_{\downarrow}$. These deviations can be expressed in terms of the numbers $\mathcal{N}_{l_{c}, l_{s}}$ of Eq. (54) as follows,

$$
\Delta N_{\uparrow}=\sum_{l_{c}= \pm 1}\left(-l_{c}\right) \mathcal{N}_{l_{c},+1} ; \quad \Delta N_{\downarrow}=\sum_{l_{c}= \pm 1}\left(-l_{c}\right) \mathcal{N}_{l_{c},-1} .
$$

Following the results of Ref. 31, let us consider the four expectation values $R_{\alpha, l_{\alpha}}=\left\langle\hat{R}_{\alpha, l_{\alpha}}\right\rangle$, where $\alpha=c, s$ and $l_{\alpha}$ $=-1,+1$, and the corresponding operator $\hat{R}_{c,-1}$ counts the number of electron doubly occupied sites, $\hat{R}_{c,+1}$ counts the number of electron empty sites, $\hat{R}_{s,-1}$ counts the number of spin-down electron singly occupied sites, and $\hat{R}_{s,+1}$ counts 
the number of spin-up electron singly occupied sites. The expressions of these operators are given in Eqs. (23) and (24) of Ref. 31. These operators obey the following relations,

$$
\begin{aligned}
& \hat{R}_{c,+1}= N_{a}-\hat{N}+\hat{R}_{c,-1} ; \quad \hat{R}_{s,-1}=\hat{N}_{\downarrow}-\hat{R}_{c,-1} ; \\
& \hat{R}_{s,+1}=\hat{N}_{\uparrow}-\hat{R}_{c,-1} .
\end{aligned}
$$

From the summation of the three relations of Eq. (56) we find the following fourth dependent relation:

$$
\sum_{\alpha=c, s} \sum_{l_{\alpha}= \pm 1} \hat{R}_{\alpha, l_{\alpha}}=N_{a}
$$

The operational relations (56) and (57) are valid for the whole parameter space. Furthermore, they reveal that at given spin $\sigma$ electron numbers, out of the four expectation values of the operators of Eq. (57) only one is independent. In the previous section we studied the double-occupation expectation value $D \equiv R_{c,-1}$. Use of the operational Eqs. (56) and (57) provides the corresponding values for $R_{c,+1}$, $R_{s,-1}$, and $R_{s,+1}$.

In the limit $U / t \rightarrow \infty$ the energy-eigenstate expectation values of the four number operators $\hat{R}_{c,-1}, \hat{R}_{c,+1}, \hat{R}_{s,-1}$, and $\hat{R}_{s,+1}$ become good quantum numbers. ${ }^{31,32}$ Thus in that limit each energy eigenstate corresponds to real-space lattice electron site distribution configurations with the same values for $R_{c,-1}, R_{c,+1}, R_{s,-1}$, and $R_{s,+1}$. For finite values of $U / t$ each energy eigenstate corresponds in general to a superposition of real-space lattice electron site distribution configurations with different numbers of electron doubly occupied sites, electron empty sites, spin-down electron singly occupied sites, and spin-up electron singly occupied sites.

Let us use the relations of Eq. (56) to evaluate expressions for the expectation values $R_{c,+1}, R_{s,-1}$, and $R_{s,+1}$ for the particular case of a ground state, from the corresponding $m$ $=0$ electron double-occupation expression defined by Eqs. (26) and (36). We find that for $m=0$, all values of $U / t$, and values of the electronic density in the domain $0 \leqslant n \leqslant 1$, these ground-state expectation values read

$$
R_{c,+1}^{0}=N_{a}-N+\frac{N}{2}\left(\frac{n}{2}\right) f(n, U / t),
$$

and

$$
R_{s,-1}^{0}=R_{s,+1}^{0}=\frac{N}{2}\left[1-\left(\frac{n}{2}\right) f(n, U / t)\right],
$$

respectively. Here $f(n, U / t)$ is the function given in Eqs. (36) and (37). Note that these ground-state expectation values change from $R_{c,-1}^{0} \equiv D_{0}=N / 2(n / 2), \quad R_{c,+1}^{0}=\left[N_{a}-N\right]$ $+N / 2(n / 2)$, and $R_{s,-1}^{0}=R_{s,+1}^{0}=N / 2[1-(n / 2)]$ as $U / t$ $\rightarrow 0$ to $R_{c,-1}^{0} \equiv D_{0}=0, R_{c,+1}^{0}=\left[N_{a}-N\right]$, and $R_{s,-1}^{0}=R_{s,+1}^{0}$ $=N / 2$ when $U / t \rightarrow \infty$, and these quantities become good quantum numbers.

Importantly, in the limit $U / t \rightarrow \infty$ the four deviations $\Delta R_{\alpha, l_{\alpha}}=\left[R_{\alpha, l_{\alpha}}-R_{\alpha, l_{\alpha}}^{0}\right]$ such that $\alpha=c, s$ and $l_{\alpha}=-1,+1$ of any eigenstate of the spin $\sigma=\uparrow, \downarrow$ electron number operators which result from application onto that state of the above general operator $\hat{O}_{\mathcal{N}}$ are restricted to the following ranges,

$$
\begin{gathered}
-\sum_{l_{s}= \pm 1} \mathcal{N}_{+1, l_{s}} \leqslant \Delta D \equiv \Delta R_{c,-1} \leqslant \sum_{l_{s}= \pm 1} \mathcal{N}_{-1, l_{s}}, \\
-\sum_{l_{s}= \pm 1} \mathcal{N}_{-1, l_{s}} \leqslant \Delta R_{c,+1} \leqslant \sum_{l_{s}= \pm 1} \mathcal{N}_{+1, l_{s}}, \\
-\sum_{l_{c}, l_{s}= \pm 1} \delta_{l_{c},-l_{s}} \mathcal{N}_{l_{c}, l_{s}} \leqslant \Delta R_{s,-1} \leqslant \sum_{l_{c}, l_{s}= \pm 1} \delta_{l_{c}, l_{s}} \mathcal{N}_{l_{c}, l_{s}},
\end{gathered}
$$

and

$$
-\sum_{l_{c}, l_{s}= \pm 1} \delta_{l_{c}, l_{s}} \mathcal{N}_{l_{c}, l_{s}} \leqslant \Delta R_{s,+1} \leqslant \sum_{l_{c}, l_{s}= \pm 1} \delta_{l_{c},-l_{s}} \mathcal{N}_{l_{c}, l_{s}},
$$

respectively. Obviously, given one of the four ranges of values defined by the inequalities (60)-(63), the other three follow from the operational relations of Eq. (56).

The maximum (and minimum) values of the inequalities (60) and (61) [and Eqs. (62) and (63)] are reached when all the electrons created by the operator $\hat{O}_{\mathcal{N}}$, in a number of $\Sigma_{l_{s}= \pm 1} \mathcal{N}_{-1, l_{s}}$, transform an equal number of electron singly occupied sites into electron doubly occupied sites. In addition, it is required that all the annihilation operators of that operator, in a number of $\Sigma_{l_{s}= \pm 1} \mathcal{N}_{+1, l_{s}}$, transform an equal number of electron singly occupied sites into electron empty sites for all the real-space lattice electron site distribution configurations of the initial state with a number of electron singly occupied sites larger than or equal to $\mathcal{N}$. Note that if the initial state includes distribution configurations whose number of electron singly occupied sites is smaller than $\mathcal{N}$, then the maximum value of $\Delta D$ is smaller than $\Sigma_{l_{s}= \pm 1} \mathcal{N}_{-1, l_{s}}$ and thus the inequalities (60) and (61) [and Eqs. (62) and (63)] are also satisfied.

The minimum (and maximum) values of the inequalities (60) and (61) [and Eqs. (62) and (63)] are reached when all the electrons created by the operator $\hat{O}_{\mathcal{N}}$, in a number of $\Sigma_{l_{s}= \pm 1} \mathcal{N}_{-1, l_{s}}$, transform an equal number of electron empty sites into electron singly occupied sites. Furthermore, it is required that all the $\Sigma_{l_{s}= \pm 1} \mathcal{N}_{+1, l_{s}}$ annihilation operators of that operator transform an equal number of electron doubly occupied sites into electron singly occupied sites for all the real-space lattice electron site distribution configurations of the initial state with a number of electron empty sites equal to or larger than $\Sigma_{l_{s}= \pm 1} \mathcal{N}_{-1, l_{s}}$ and a number of electron doubly occupied sites equal to or larger than $\Sigma_{l_{s}= \pm 1} \mathcal{N}_{+1, l_{s}}$. Note that if the initial state includes distribution configurations whose number of electron empty sites is smaller than $\Sigma_{l_{s}= \pm 1} \mathcal{N}_{-1, l_{s}}$ and/or whose number of electron doubly occupied sites is smaller than $\Sigma_{l_{s}= \pm 1} \mathcal{N}_{+1, l_{s}}$, the minimum value 
of $\Delta D$ is larger than $-\Sigma_{l_{s}= \pm 1} \mathcal{N}_{+1, l_{s}}$ and thus the inequalities (60) and (61) [and Eqs. (62) and (63)] are also satisfied.

It is found in Ref. 32 that the $U / t \rightarrow \infty$ electron doubleoccupation selection rules associated with the inequalities (60)-(63) are related to the occurrence of other selection rules which concern the range of the deviations in the number of $\pm 1 / 2$ holons and $\pm 1 / 2$ spinons. The holon and spinon selection rules are valid for all values of $U / t$ and play an important role in the evaluation of correlation functions. ${ }^{32,36,37}$ These selection rules are exact in the case of rotated-electron operators. In the case of the corresponding electron operators such rules provide the numbers of holons and spinons of the final states which contribute most significantly to the few-electron correlation functions. ${ }^{32}$ This relationship between the $U / t \rightarrow \infty$ electron double-occupation deviations and the deviations in the number $\pm 1 / 2$ holons and $\pm 1 / 2$ spinons follows from the unitary transformation which maps electrons onto rotated electrons. This transformation also maps the electron double-occupation operator onto an operator that counts the number of $-1 / 2$ holons. The latter operator is nothing but the rotated-electron doubleoccupation operator. ${ }^{32}$

\section{THE PSEUDOPARTICLE ENERGY BANDS}

In Sec. IV we found that the pseudoparticle energy bands defined by Eqs. (C15)-(C21) of Ref. 31 play an important role in the electron double-occupation spectra of the pseudoparticles, as confirmed by Eq. (41). Moreover, in Ref. 37 it was found that the shape of the lines in the frequency/ energy and momentum planes where the peaks or edges in the one-electron and two-electron spectral-weight distributions are located correspond to the pseudoparticle energy bands. Thus the study of the band-momentum dependence of these energy bands is a problem of interest for understanding the one-electron and two-electron spectral properties of the quantum liquid. The $c$-pseudoparticle and $s, 1$-pseudoparticle energy bands were studied and plotted in Ref. 51 for finite values of the spin density. On the other hand, the $c, \nu$-pseudoparticle and $s, \nu$-pseudoparticle energy bands for $\nu>1$ were first introduced in Ref. 38, yet these bands were not plotted in that reference. Since most studies on the finiteenergy spectral properties of low-dimensional materials refer to zero magnetization, it is worthwhile plotting and discussing the band-momentum dependence of the above pseudoparticle bands for zero-spin density.

The general excitation energy spectrum of interest for the problem of the finite-energy correlation functions is given in Eq. (24). The band-momentum-independent term $\omega_{0}$ is defined in Eq. (25). The value of this finite energy is determined by the holon and spinon deviations and corresponds to the finite-energy edges of the correlation functions. The other term of the energy spectrum (24) is of gapless character and in the case of zero magnetization involves the pseudoparticle energy bands $\epsilon_{c}(q), \quad \epsilon_{s, 1}(q)$, and $\epsilon_{c, \nu}^{0}(q)$ where $\nu$ $=1,2,3, \ldots$. These bands are defined in Eqs. $(\mathrm{C} 15)-(\mathrm{C} 17)$ of Ref. 31. As discussed below, at zero magnetization both the energy and band-momentum width of the energy bands $\epsilon_{s, \nu}^{0}(q)$ vanish in the case of the $\nu>1$ branches. According to Eqs. (C1)-(C3) of Ref. 31, in the case of the zero-spindensity ground states the band $\epsilon_{c}(q)$ is filled (and empty) for band-momentum values such that $|q| \leqslant 2 k_{F}$ (and $2 k_{F}<|q|$ $\leqslant \pi$ ). On the other hand, for such ground states the band $\boldsymbol{\epsilon}_{s, 1}(q)$ is full and the bands $\epsilon_{c, \nu}^{0}(q)$ where $\nu=1,2,3, \ldots$ are empty. The expressions of the bands $\epsilon_{c}(q), \epsilon_{s, 1}(q), \epsilon_{c, 1}^{0}(q)$, and $\epsilon_{c, 2}^{0}(q)$ are plotted in Figs. 6-9 as a function of the band momentum $q$ for spin density $m=0$, different values of $U / t$, and of the electronic density $n$.

As is confirmed by analysis of the energy-band curves plotted in Figs. 6(a) and 6(b), both the energy and bandmomentum width of the $c$-pseudoparticle bands are density, spin-density, and $U / t$ independent and given by $4 t$ and $2 \pi$, respectively. From the $U / t$ dependence of the curves of these figures, we find that the main effect of increasing the on-site repulsion $U / t$ is the increasing of the energy-band-width of the ground-state filled sea and the corresponding decreasing of the band-energy width of the corresponding unfilled region. The main effect of changing the electronic density is on the ground-state $c$-pseudoparticle Fermi points, which are given by $\pm 2 k_{F}$.

In contrast to the energy and band-momentum widths of the $c$ pseudoparticles, the energy width of the $\alpha, \nu$ pseudoparticle bands plotted in Figs. 7-9 is a decreasing function of $U / t$, vanishing in the limit $U / t \rightarrow \infty$. This property is a result of the fact that all $\eta$-spin and spin configurations are degenerated in that limit. The band-momentum width of the $c, \nu$-pseudoparticle bands and of the $s, \nu$-pseudoparticle bands belong to branches such that $\nu$ $>1$ are density and spin-density dependent, respectively, and are given by $2\left[\pi-2 k_{F}\right]$ and $2\left[k_{F \uparrow}-k_{F \downarrow}\right]$, respectively. Note also that the $\alpha, \nu$-pseudoparticle energy bands $\epsilon_{s, 1}^{0}(q)$ $=\epsilon_{s, 1}(q)-2 \mu_{0} H$ and $\epsilon_{\alpha, \nu}^{0}(q)$ vanish at the bandmomentum limiting values, i.e.,

$$
\epsilon_{\alpha, \nu}^{0}\left( \pm q_{\alpha, \nu}\right)=0
$$

where according to Eqs. (C12)-(C14) of Ref. 31, $q_{c, \nu}=[\pi$ $\left.-2 k_{F}\right], q_{s, 1}=k_{F \uparrow}$, and $q_{s, \nu}=\left[k_{F \uparrow}-k_{F \downarrow}\right]$ for the $\nu>1$ branches.

For finite values of the spin density $m$ and magnetic field $H$, the $s, \nu$-pseudoparticle energy bands $\epsilon_{s, \nu}^{0}(q)$ have for the $\nu>1$ branches both finite momentum width, given by the above expression $2\left[k_{F \uparrow}-k_{F \uparrow}\right]$, and finite energy width, given by $\left|\epsilon_{s, \nu}^{0}(0)\right|$. The energy-band value at $q=0$ is negative, i.e., $\epsilon_{s, \nu}^{0}(0) \leqslant 0$. As the spin density $m \rightarrow 0$, both widths $2\left[k_{F \uparrow}-k_{F \uparrow}\right] \rightarrow 0$ and $\left|\epsilon_{s, \nu}^{0}(0)\right| \rightarrow 0$ vanish. Thus, for the $\nu$ $>1$ branches the $s, \nu$-pseudoparticle energy bands collapse into the point $\left\{q=0, \epsilon_{s, \nu}^{0}(0)=0\right\}$ in that limit. Since Figs. 6-9 correspond to $m=0$, these bands are not plotted in these figures. The finite-magnetization case will be considered elsewhere.

The $c, \nu$-pseudoparticle bands plotted in Figs. 8(a), 8(b), 9(a), and 9(b) have their maximum value at $q=0$. These 

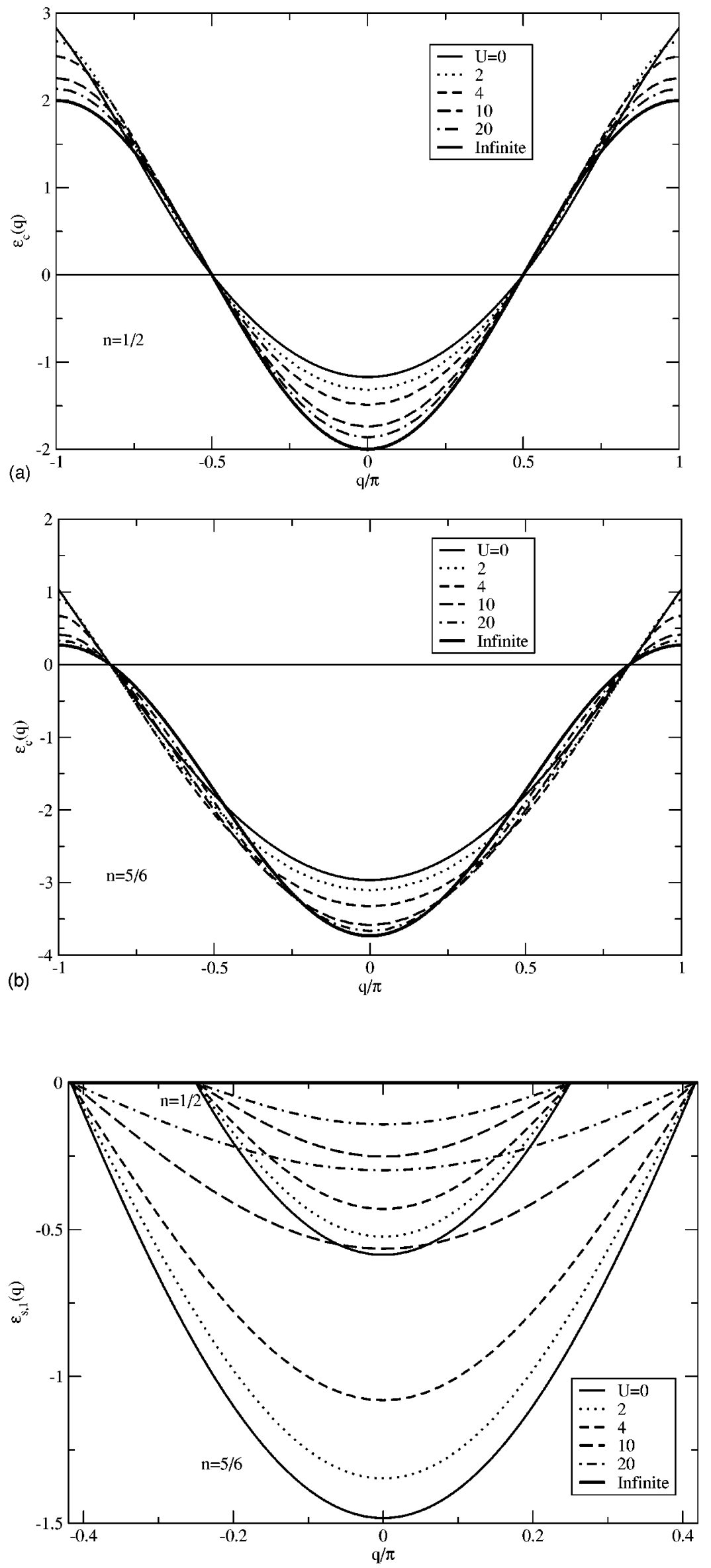

FIG. 6. The pseudoparticle energy band $\epsilon_{c}(q)$ in units of $t$ plotted for electronic density (a) $n$ $=1 / 2$ and (b) $n=5 / 6$ and on-site repulsion values $U / t \rightarrow 0, \quad U / t=2.0, \quad U / t=4.0, \quad U / t=10, \quad U / t$ $=20$, and $U / t \rightarrow \infty$. The ground-state Fermi level corresponds to zero energy and is marked by a horizontal line which overlaps the $\epsilon_{c}(q)$ band at the band-momentum Fermi points $q= \pm 2 k_{F}$ $= \pm \pi / 2$.
FIG. 7. The pseudoparticle energy band $\epsilon_{s, 1}(q)$ in units of $t$ plotted for electronic densities $n=1 / 2$ and $n=5 / 6$ and on-site repulsion values $U / t \rightarrow 0, U / t=2.0, U / t=4.0, U / t=10, U / t$ $=20$, and $U / t \rightarrow \infty$. The ground-state Fermi level corresponds to zero energy and overlaps the energy band at the band-momentum Fermi points $q= \pm k_{F}$. 

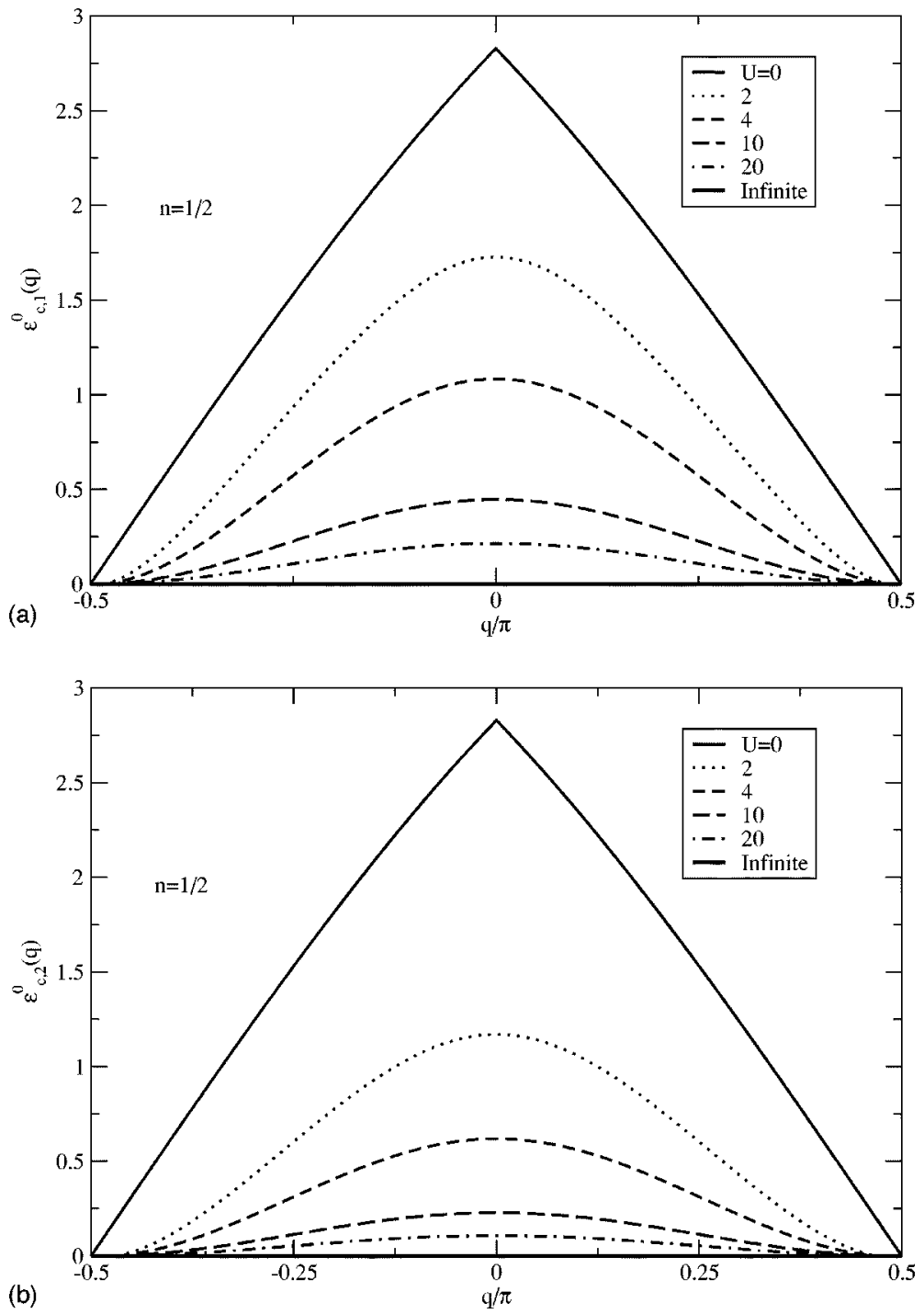

FIG. 8. The pseudoparticle energy bands (a) $\epsilon_{c, 1}^{0}(q)$ and (b) $\epsilon_{c, 2}^{0}(q)$ in units of $t$ plotted for electronic density $n=1 / 2$ and on-site repulsion values $U / t \rightarrow 0, U / t=2.0, U / t=4.0, U / t=10$, and $U / t=20$. The $U / t \rightarrow \infty$ limit of these bands corresponds to a horizontal line located at the zero energy level. bands are thus inverted. As discussed below, as the electronic density approaches 1 , both the energy and momentum widths of these bands vanish. Except for the case of the $s, 1$ the vicinity of the band-momentum limiting values $q= \pm q_{c}$ $= \pm \pi$ and $q= \pm q_{c, \nu}= \pm\left[\pi-2 k_{F}\right]$. In contrast, note that the $s, 1$ band has a linear $q$ dependence in the vicinity of the band-momentum limiting values $q= \pm q_{s, 1}= \pm k_{F}$. On the other hand, for finite values of $U / t$ all pseudoparticle bands show a quadratic dependence on the band momentum $q$ in the vicinity of the point $q=0$, as confirmed by the curves of Figs. 6-9.

From analysis of Figs. 8 and 9, we find that the $\epsilon_{c, 1}^{0}(q)$ and $\epsilon_{c, 2}^{0}(q)$ bands vanish at the band-momentum limiting values $q= \pm q_{c, \nu}= \pm\left[\pi-2 k_{F}\right]$. This behavior also occurs for finite values of the spin density $m$. For such values also the $\epsilon_{s, \nu}^{0}(q)$ bands vanish for $q= \pm q_{s, \nu}= \pm\left[k_{F \uparrow}-k_{F \downarrow}\right]$. The energy band $\epsilon_{c, \nu}^{0}(q)$ has its maximum value at $q=0$, with $\epsilon_{c, \nu}^{0}(0) \geqslant 0$, as shown in Figs. 8(a), 8(b), and 9(a), 9(b). The energy band $\epsilon_{s, \nu}^{0}(q)$, for spin density $m>0$, has its minimum at $q=0$, with $\epsilon_{s, \nu}^{0}(0) \leqslant 0$, as in the case of the $s, 1$ energy band plotted in Fig. 7 for $m=0$.

In the Appendix we consider the limiting expressions of the pseudoparticle energy bands in the case of zero-spin density $m=0$. Note that according to the expressions given in Eqs. (A1)-(A3) of the Appendix, the $q$ dependence of the $c$-pseudoparticle energy band in the vicinity of $q= \pm \pi$ and of the $c, \nu$ bands in the vicinity of $q=0$ is different for finite values of $U / t$ and in the limit $U / t \rightarrow 0$. As mentioned above, for finite values of $U / t$ the dependence on the band momentum $q$ is quadratic in the vicinity of these band-momentum values. Thus in that case the $q$ derivative of these bands vanishes both at $q= \pm \pi$ and $q=0$ respectively, as confirmed by the curves of Figs. 6-9 and by the expressions of Eqs. (A1) and (A3) of the Appendix. In contrast, analysis of the data of these figures and equations reveals that in the limit $U / t \rightarrow 0$ the $q$ derivative at these band-momentum values becomes finite and the $q$ dependence in their vicinity is linear instead of quadratic. This singular change results from the nonperturbative character of the electronic correlations which leads to a different physics at $U / t=0$ and for $U / t$ $>0$. In the particular case of half filling, this effect occurs for the $c$-pseudoparticle band at the band-momentum Fermi points $q= \pm \pi$. In this case, that effect is associated with the band of Fig. 7, all remaining bands plotted in Figs. 6-9 show 

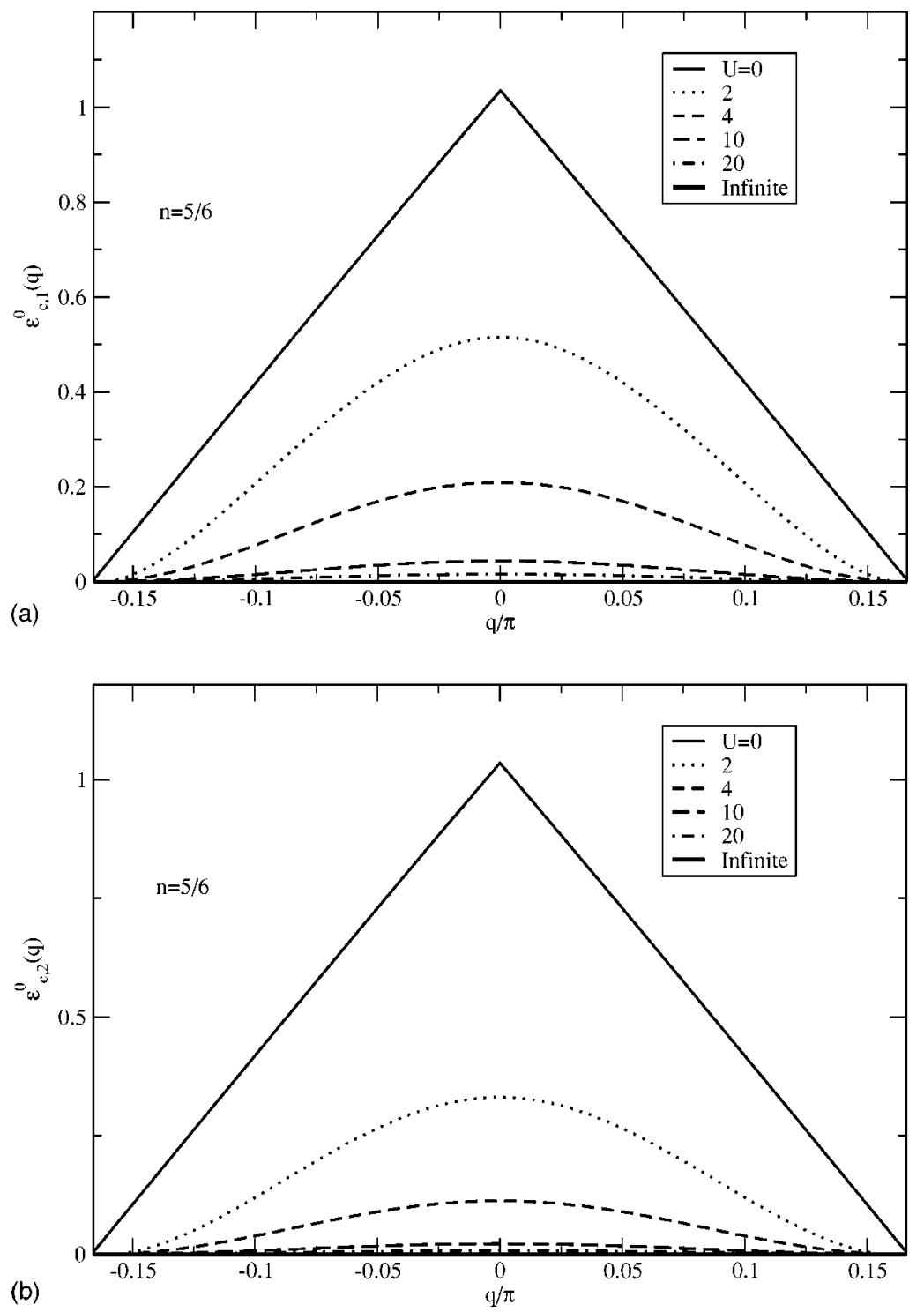

FIG. 9. The pseudoparticle energy bands (a) $\epsilon_{c, 1}^{0}(q)$ and (b) $\epsilon_{c, 2}^{0}(q)$ in units of $t$ plotted for electronic density $n=5 / 6$ and on-site repulsion values $U / t \rightarrow 0, U / t=2.0, U / t=4.0, U / t=10$, and $U / t=20$. The $U / t \rightarrow \infty$ limit of these bands corresponds to a horizontal line located at the zero energy level. for finite values of $U / t$ a quadratic dependence on $q$ in singular Mott-Hubbard insulator-metal transition. For other electronic densities this effect occurs at unoccupied groundstate band-momentum values and therefore, is not associated with such a transition. The vanishing at half filling of the band $\epsilon_{c, \nu}^{0}(q)$ of Eq. (A6) of the Appendix results from its collapse into the point $\left\{q=0, \epsilon_{c, \nu}^{0}(0)=0\right\}$ as $\delta \rightarrow 0$, where $\delta=(1-n)$ is the doping concentration. This is confirmed by the dependence on $n$ (and $\delta$ ) of expressions (A3) for that band. We emphasize that both the $s, \nu$ band for the $\nu>1$ branches and the $c, \nu$ band collapse into the point $\{q$ $\left.=0, \epsilon_{\alpha, \nu}^{0}(0)=0\right\}$ with $\alpha=s$ and $\alpha=c$, respectively, as the spin density $m \rightarrow 0$ and the doping $\delta=(1-n) \rightarrow 0$, respectively.

The terms of the Landau energy functional given in Eq. (24) describe the energy spectra of all one-electron and twoelectron elementary excitations of the 1D Hubbard model. ${ }^{31}$ Within such a functional description, each elementary excitation is simply described by specific values of the pseudoparticle band-momentum distribution function devia- tions defined in Eq. (58) of Ref. 31. These deviations describe (i) pseudoparticle-pseudoparticle hole processes in the $c$ and $s, 1$ bands which conserve the pseudoparticle numbers; (ii) creation or annihilation of $c$ and $s, 1$ pseudoparticles; (iii) creation of $c, \nu$ pseudoparticles and of $s, \nu$ pseudoparticles with $\nu>1$; and (iv) creation of $-1 / 2$ Yang holons and of $-1 / 2$ LH spinons.

All the energy spectra obtained previously in the literature, such as, for instance, the ones studied in the Refs. 4146 , can be expressed in terms of the pseudoparticle energy bands plotted for $m=0$ in Figs. 6-9. The authors of these studies used for each specific elementary excitation different forms for the two Lieb and $\mathrm{Wu}$ equations ${ }^{21}$ or/and for the thermodynamic Takahashi's equations ${ }^{22}$ provided in Ref. 31. On the other hand, insertion in the energy Landau-liquid functional (24) of band-momentum distribution function deviations suitable to these excitations leads to the same energy spectra. In addition, the energy functional (24) provides the spectrum for all values of the energy of all excitations which contribute to the one-electron and two-electron physics, as mentioned above. 


\section{CONCLUDING REMARKS}

In this paper we studied the electron double-occupation deviations which result from creation of $c$ pseudoparticles; $\alpha, \nu$ pseudoparticles; $-1 / 2$ Yang holons; and $-1 / 2 \mathrm{HL}$ spinons, where $\alpha=c, s$ and $\nu=1,2,3, \ldots$. All energy eigenstates can be described in terms of occupancy configurations of these quantum objects which are related to electrons and rotated electrons in Refs. 31 and 32. Here we introduced an electron double-occupation functional whose coefficients are pseudoparticle band-momentum-dependent doubleoccupation spectra. Our investigations provided interesting information about the electron site distribution configurations of the real-space lattice which describe the energy eigenstates. The study of these spectra provided important information on the localization/delocalization degree of the real-space lattice electron site distribution configurations which describe the elementary quantum objects. We found that for some of the pseudoparticle branches such a degree of localization/delocalization is strongly dependent on the value of the band momentum. We also considered $U / t \rightarrow \infty$ selection rules, which limit the ranges of the electron doubleoccupation deviations that result from excitations generated by $\mathcal{N}$-electron physical operators. These rules are the starting point for finding related selection rules, which are investigated elsewhere. ${ }^{32}$ The latter rules are valid for all values of $U / t$ and play an important role in the study of finite-energy correlation functions.

This paper is a first step in the use of the general holon and spinon scenarios in the study of the finite-energy spectral properties of the 1D Hubbard model. In addition to the introduction and study of the electron double-occupation functional, in this paper we studied the dependence on the band momentum $q$, on-site repulsion $U / t$, and electronic density $n$ of the pseudoparticle energy bands associated with such a functional. As was mentioned in this paper, these bands play a central role in the spectral properties of the model. ${ }^{37}$

The holon and spinon descriptions of the 1D Hubbard model introduced in Ref. 31 and further studied here are used in Ref. 32 for finding further useful microscopic information on the spectral properties of the model. By combining the results obtained here with that information, one can evaluate expressions for finite-energy one-electron and two-electron correlation functions. $^{31,36,37}$ That is a problem of major importance for the study of the unusual finite-energy spectral properties observed in real quasi-one-dimensional materials. ${ }^{1-20}$ Elsewhere it is confirmed that the holon and spinon descriptions of the energy eigenstates introduced in Ref. 31 and further developed here and in related papers $^{31,32,36,37}$ are suitable for the successful study of these finite-energy spectral properties.

\section{ACKNOWLEDGMENTS}

We thank Jim W. Allen, Daniel Bozi, Antônio Castro Neto, Ralph Claessen, Francisco (Paco) Guinea, Katrina Hibberd, Peter Horsch, João M. B. Lopes dos Santos, Luís M. Martelo, Karlo Penc, and J. M. Román for stimulating discussions.

\section{APPENDIX: LIMITING EXPRESSIONS OF THE PSEUDOPARTICLE ENERGY BANDS}

In this Appendix we present the limiting expressions of the pseudoparticle energy bands in the case of zero-spin density $m=0$. In the limits $U / t \rightarrow 0$ and $U / t \gg 1$ one can evaluate closed-form expressions for the bands $\epsilon_{c}(q), \epsilon_{s, 1}(q)$, and $\epsilon_{\alpha, \nu}^{0}(q)$ where $\alpha=c, s$ and $\nu=1,2,3, \ldots$, defined in Appendix $\mathrm{C}$ of Ref. 31. The simplest case is that of the $s, \nu$ energy bands for the $\nu>1$ branches, which at zero-spin density $m=0$ are given for all values of $U / t$ and $n$ by $\epsilon_{s, \nu}^{0}(q)$ $=0$ for $|q| \leqslant\left(k_{F \uparrow}-k_{F \downarrow}\right)=0$. As $m \rightarrow 0$ these $\nu>1$ bands collapse in that limit into the point $\left\{q=0, \epsilon_{s, \nu}^{0}(0)=0\right\}$. The expressions of the remaining pseudoparticle bands for zerospin density $m=0$, values of the density $0 \leqslant n \leqslant 1$, and $U / t$ $\rightarrow 0$ and $U / t \gg 1$ are the following,

$$
\begin{aligned}
& \epsilon_{c}(q)=-4 t\left[\cos \left(\frac{q}{2}\right)-\cos \left(\frac{\pi n}{2}\right)\right] ; \quad|q| \leqslant 2 k_{F}, \quad U / t \rightarrow 0 \\
& =-2 t\left[\cos \left(|q|-\frac{\pi n}{2}\right)-\cos \left(\frac{\pi n}{2}\right)\right] \text {; } \\
& 2 k_{F} \leqslant|q| \leqslant \pi, \quad U / t \rightarrow 0 ; \\
& =-2 t[\cos (q)-\cos (\pi n)] \\
& -\frac{(2 t)^{2}}{U} 2 n \ln (2)\left[\sin ^{2}(q)-\sin ^{2}(\pi n)\right] ; \\
& |q| \leqslant \pi, \quad U / t \gg 1, \\
& \epsilon_{s, 1}(q)=-2 t\left[\cos (q)-\cos \left(\frac{\pi n}{2}\right)\right] ; \quad|q| \leqslant k_{F}, \quad U / t \rightarrow 0 \\
& =-\frac{(2 t)^{2}}{U} \frac{\pi}{2}\left[n-\frac{\sin (2 \pi n)}{2 \pi}\right] \cos \left(\frac{q}{n}\right) \\
& |q| \leqslant k_{F}, \quad U / t \gg 1, \\
& \epsilon_{c, \nu}^{0}(q)=4 t \cos \left(\frac{|q|+\pi n}{2}\right) ; \quad|q| \leqslant\left(\pi-2 k_{F}\right), \quad U / t \rightarrow 0 ; \\
& =\frac{(4 t)^{2}}{U} \frac{1}{2 \nu}\left[\delta-\frac{\sin (2 \pi \delta)}{2 \pi}\right] \cos ^{2}\left(\frac{q}{2 \delta}\right) \text {; } \\
& |q| \leqslant\left(\pi-2 k_{F}\right), \quad U / t \gg 1,
\end{aligned}
$$

where $\delta \equiv(1-n)$ is the doping concentration. The expressions (A1) and (A2) were already obtained in the $c$ and $s$ $\equiv s, 1$ pseudoparticle studies of Ref. 51. From analysis of the band momentum $q$, on-site repulsion $U / t$, and density $n$ dependence of the band expressions (A1)-(A3), we find that these agree with the corresponding dependence on the same parameters of the curves of Figs. 6-9. 
At half filling and spin density $m=0$ the integral equations which define the ground-state rapidities and pseudoparticle bands can be solved by Fourier transform. Thus in this case one can derive the following expressions for the pseudoparticle bands, which are valid for all values of $U / t$ $>0,{ }^{52}$

$$
\begin{aligned}
\epsilon_{c}(q)= & -2 t\left[\cos k^{0}(q)+1\right] \\
& -4 t \int_{0}^{\infty} d x J_{1}(x) \frac{\cos \left[x \sin k^{0}(q)\right]}{x\left[1+e^{x U / 2 t}\right]} ;|q| \leqslant \pi,
\end{aligned}
$$

$$
\epsilon_{s, 1}(q)=-2 t \int_{0}^{\infty} d x J_{1}(x) \frac{\cos \left[x \Lambda_{s, 1}^{0}(q)\right]}{x \cosh \left(\frac{x U}{4 t}\right)} ; \quad|q| \leqslant \pi / 2,
$$

and

$$
\epsilon_{c, \nu}^{0}(q)=0 ; \quad|q| \leqslant\left(\pi-2 k_{F}\right)=0 .
$$

The inverse functions of the half filling ground-state rapidity-momentum function $k^{0}(q)$ and rapidity function $\Lambda_{s, 1}^{0}(q)$ involved in expressions (A4) and (A5) are given in Eqs. (33) and (34), respectively.
${ }^{1}$ N. E. Hussey, M. N. McBrien, L. Balicas, J. S. Brooks, S. Horii, and H. Ikuta, Phys. Rev. Lett. 89, 086601 (2002).

${ }^{2}$ A. Menzel, R. Beer, and E. Bertel, Phys. Rev. Lett. 89, 076803 (2002).

${ }^{3}$ Shin-ichi Fujimori, Akihiro Ino, Testuo Okane, Atsushi Fujimori, Kozo Okada, Toshio Manabe, Masahiro Yamashita, Hideo Kishida, and Hiroshi Okamoto, Phys. Rev. Lett. 88, 247601 (2002).

${ }^{4}$ M. Z. Hasan, P. A. Montano, E. D. Isaacs, Z.-X. Shen, H. Eisaki, S. K. Sinha, Z. Islam, N. Motoyama, and S. Uchida, Phys. Rev. Lett. 88, 177403 (2002).

${ }^{5}$ R. Claessen, M. Sing, U. Schwingenschlögl, P. Blaha, M. Dressel, and C. S. Jacobsen, Phys. Rev. Lett. 88, 096402 (2002).

${ }^{6}$ G.-H. Gweon, J. D. Denlinger, C. G. Olson, H. Hochst, J. Marcus, and C. Schlenker, Physica B 312-313, 584 (2002); G.-H. Gweon, J. D. Denlinger, J. W. Allen, R. Claessen, C. G. Olson, H. Hochst, J. Marcus, C. Schlenker, and L. F. Schneemeyer, J. Electron Spectrosc. Relat. Phenom. 117-118, 481 (2001).

${ }^{7}$ P. Monceau, F. Ya. Nad, and S. Brazovskii, Phys. Rev. Lett. 86, 4080 (2001).

${ }^{8}$ K. Takenaka, K. Nakada, A. Osuka, S. Horii, H. Ikuta, I. Hirabayashi, S. Sugai, and U. Mizutani, Phys. Rev. Lett. 85, 5428 (2000).

${ }^{9}$ T. Mizokawa, C. Kim, Z.-X. Shen, A. Ino, T. Yoshida, A. Fujimori, M. Goto, H. Eisaki, S. Uchida, M. Tagami, K. Yoshida, A. I. Rykov, Y. Siohara, K. Tomimoto, S. Tajima, Yuh Yamada, S. Horii, N. Yamada, Yasuji Yamada, and I. Hirabayashi, Phys. Rev. Lett. 85, 4779 (2000).

${ }^{10}$ J. Moser, J. R. Cooper, D. Jérome, B. Alavi, S. E. Brown, and K. Bechgaard, Phys. Rev. Lett. 84, 2674 (2000).

${ }^{11}$ G. Mihály, I. Kézsmárki, F. Zámborszky, and L. Forró, Phys. Rev. Lett. 84, 2670 (2000).

${ }^{12}$ V. Vescoli, F. Zwick, J. Voit, H. Berger, M. Zacchigna, L. Degiorgi, M. Grioni, and G. Grüner, Phys. Rev. Lett. 84, 1272 (2000).

${ }^{13}$ J. D. Denlinger, G. H. Gweon, J. W. Allen, C. G. Olson, J. Marcus, C. Schlenker, and L.-S. Hsu, Phys. Rev. Lett. 82, 2540 (1999).

${ }^{14}$ H. Fujisawa, T. Yokoya, T. Takahashi, S. Miyasaka, M. Kibune, and H. Takagi, Phys. Rev. B 59, 7358 (1999).

${ }^{15}$ K. Kobayashi, T. Mizokawa, A. Fujimori, M. Isobe, Y. Ueda, T. Tohyama, and S. Maekawa, Phys. Rev. Lett. 82, 803 (1999); K.
Kobayashi, T. Mizokawa, and A. Fujimori, ibid. 80, 3121 (1998).

${ }^{16}$ Claude Bourbonnais and Denis Jérome, Science (Washington, DC, U.S.) 281, 1155 (1998).

${ }^{17}$ V. Vescoli, L. Degiorgi, W. Henderson, G. Grüner, K. P.Starkey, and L. K. Montgomery, Science (Washington, DC, U.S.) 281, 1181 (1998).

${ }^{18}$ F. Zwick, D. Jérome, G. Margaritondo, M. Onellion, J. Voit, and M. Grioni, Phys. Rev. Lett. 81, 2974 (1998); F. Zwick, S. Brown, G. Margaritondo, C. Merlic, M. Onellion, J. Voit, and M. Grioni, ibid. 79, 3982 (1997).

${ }^{19}$ T. Mori, T. Kawamoto, J. Yamaura, T. Enoki, Y. Misaki, T. Yamabe, H. Mori, and S. Tanaka, Phys. Rev. Lett. 79, 1702 (1997).

${ }^{20}$ C. Kim, A. Y. Matsuura, Z.-X. Shen, N. Motoyama, H. Eisaki, S. Uchida, T. Tohyama, and S. Maekawa, Phys. Rev. Lett. 77, 4054 (1996); N. Motoyama, H. Eisaki, and S. Uchida, Phys. Rev. Lett. 76, 3212 (1996).

${ }^{21}$ Elliott H. Lieb and F. Y. Wu, Phys. Rev. Lett. 20, 1445 (1968).

${ }^{22}$ M. Takahashi, Prog. Theor. Phys. 47, 69 (1972).

${ }^{23}$ P. B. Ramos and M. J. Martins, J. Phys. A 30, L195 (1997); M. J. Martins and P. B. Ramos, Nucl. Phys. B 522, 413 (1998).

${ }^{24}$ M. Rasetti, The Hubbard Model, Recent Results (Word Scientific, Singapore, 1991); A. Montorsi, The Hubbard Model (Word Scientific, Singapore, 1992).

${ }^{25}$ The Hubbard Model-Its Physics and Mathematical Physics, Vol. 343 of NATO Advanced Studies Institute, Series B-Physics, edited by Dionys Baeriswyl, David K. Cambell, José M. P. Carmelo, Francisco Guinea, and Enrique Louis (Plenum, New York, 1995).

${ }^{26}$ M. Granath, V. Oganesyan, D. Orgad, and S. A. Kivelson, Phys. Rev. B 65, 184501 (2002).

${ }^{27}$ D. Orgad, S. A. Kivelson, E. W. Carlson, V. J. Emery, X. J. Zhou, and Z. X. Shen, Phys. Rev. Lett. 86, 4362 (2001).

${ }^{28}$ E. W. Carlson, D. Orgad, S. A. Kivelson, and V. J. Emery, Phys. Rev. B 62, 3422 (2000).

${ }^{29}$ J. Zaanen, O. Y. Osman, H. V. Kruis, Z. Nussinov, and J. Tworzydlo, Philos. Mag. B 81, 1485 (2001).

${ }^{30}$ A. L. Chernyshev, S. R. White, and A. H. Castro Neto, Phys. Rev. B 65, 214527 (2002).

${ }^{31}$ J. M. P. Carmelo and J. M. Román, cond-mat/0302044 (unpublished).

${ }^{32}$ J. M. P. Carmelo and K. Penc, cond-mat/0303279 (unpublished). 
${ }^{33}$ A. Brooks Harris and Robert V. Lange, Phys. Rev. 157, 295 (1967).

${ }^{34}$ D. Baeriswyl, J. Carmelo, and A. Luther, Phys. Rev. B 33, 7247 (1986).

${ }^{35}$ José Carmelo and Dionys Baeriswyl, Phys. Rev. B 37, 7541 (1988).

${ }^{36}$ J. M. P. Carmelo and L. M. Martelo, and P. D. Sacramento (unpublished).

${ }^{37}$ J. M. P. Carmelo and K. Penc (unpublished).

${ }^{38}$ J. M. P. Carmelo and N. M. R. Peres, Phys. Rev. B 56, 3717 (1997).

${ }^{39}$ D. Pines and P. Nozières, in The Theory of Quantum Liquids (Addison-Wesley, Reading, MA, 1966), Vol. I.

${ }^{40}$ Gordon Baym and Christopher J. Pethick, in Landau FermiLiquid Theory Concepts and Applications (Wiley, New York, 1991).

${ }^{41}$ A. A. Ovchinnikov, Zh. Éksp. Teor. Fiz. 57, 2137 (1969) [Sov. Phys. JETP 30, 1160 (1970)].

${ }^{42}$ C. F. Coll, III, Phys. Rev. B 9, 2150 (1974).

${ }^{43}$ F. Woynarovich, J. Phys. C 15, 85 (1982); 15, 97 (1982); 16, 5293 (1983); 16, 6593 (1983).

${ }^{44}$ T. C. Choy and W. Young, J. Phys. C 15, 521 (1982).

${ }^{45}$ A. Klümper, A. Schadschneider, and J. Zittartz, Z. Phys. B: Con- dens. Matter 78, 99 (1990).

${ }^{46}$ T. Deguchi, F. H. L. Essler, F. Gölmann, A. Klümper, V. E. Korepin, and K. Kusakabe, Phys. Rep. 331, 197 (2000).

${ }^{47}$ J. M. P. Carmelo, N. M. R. Peres, and P. D. Sacramento, Phys. Rev. Lett. 84, 4673 (2000).

${ }^{48}$ O. J. Heilmann and E. H. Lieb, Ann. N.Y. Acad. Sci. 172, 583 (1971); E. H. Lieb, Phys. Rev. Lett. 62, 1201 (1989).

${ }^{49}$ C. N. Yang, Phys. Rev. Lett. 63, 2144 (1989).

${ }^{50}$ Fabian H. L. Essler, Vladimir E. Korepin, and Kareljan Schoutens, Phys. Rev. Lett. 67, 3848 (1991); Nucl. Phys. B 372, 559 (1992)

${ }^{51}$ J. Carmelo, P. Horsch, P.-A. Bares, and A. A. Ovchinnikov, Phys. Rev. B 44, 9967 (1991).

${ }^{52}$ J. M. P. Carmelo, P. Horsch, and A. A. Ovchinnikov, Phys. Rev. B 45, 7899 (1992); 46, 14728 (1992).

${ }^{53}$ J. M. P. Carmelo, J. M. E. Guerra, J. M. B. Lopes dos Santos, and A. H. Castro Neto, Phys. Rev. Lett. 83, 3892 (1999).

${ }^{54}$ Masao Ogata and Hiroyuki Shiba, Phys. Rev. B 41, 2326 (1990).

${ }^{55}$ R. G. Dias and J. M. Lopes dos Santos, J. Phys. I 2, 1889 (1992).

${ }^{56}$ Karlo Penc, Frédéric Mila, and Hiroyuki Shiba, Phys. Rev. Lett. 75, 894 (1995).

${ }^{57}$ Karlo Penc, Karen Hallberg, Frédéric Mila, and Hiroyuki Shiba, Phys. Rev. Lett. 77, 1390 (1996). 